ARTICLE

DOI: $10.1038 / s 41467-018-03544-x$

\title{
Structure of Schlafen13 reveals a new class of tRNA/rRNA- targeting RNase engaged in translational control
}

Jin-Yu Yang (10 1, Xiang-Yu Deng ${ }^{2}$, Yi-Sheng Li ${ }^{3,4}$, Xian-Cai Ma ${ }^{5}$, Jian-Xiong Feng ${ }^{1}$, Bing Yu', Yang Chen ${ }^{1,7}$, Yi-Ling Luo ${ }^{1}$, Xi Wang (1) ${ }^{3,8}$, Mei-Ling Chen ${ }^{1}$, Zhi-Xin Fang ${ }^{1}$, Fu-Xiang Zheng ${ }^{5}$, Yi-Ping Li ${ }^{5}$, Qian Zhong ${ }^{1}$, Tie-Bang Kang ${ }^{1}$, Li-Bing Song ${ }^{1}$, Rui-Hua Xu1, Mu-Sheng Zeng (1) 1, Wei Chen ${ }^{4,6}$, Hui Zhang ${ }^{5}$, Wei Xie (1) ${ }^{2} \&$ Song Gao (1) ${ }^{1}$

Cleavage of transfer ( $t$ )RNA and ribosomal ( $r$ )RNA are critical and conserved steps of translational control for cells to overcome varied environmental stresses. However, enzymes that are responsible for this event have not been fully identified in high eukaryotes. Here, we report a mammalian tRNA/rRNA-targeting endoribonuclease: SLFN13, a member of the Schlafen family. Structural study reveals a unique pseudo-dimeric U-pillow-shaped architecture of the SLFN13 $\mathrm{N}^{\prime}$-domain that may clamp base-paired RNAs. SLFN13 is able to digest tRNAs and rRNAs in vitro, and the endonucleolytic cleavage dissevers 11 nucleotides from the 3 -terminus of tRNA at the acceptor stem. The cytoplasmically localised SLFN13 inhibits protein synthesis in 293T cells. Moreover, SLFN13 restricts HIV replication in a nucleolytic activity-dependent manner. According to these observations, we term SLFN13 RNase S13. Our study provides insights into the modulation of translational machinery in high eukaryotes, and sheds light on the functional mechanisms of the Schlafen family.

\footnotetext{
${ }^{1}$ State Key Laboratory of Oncology in South China, Collaborative Innovation Center for Cancer Medicine, Sun Yat-Sen University Cancer Center, Guangzhou 510060, Guangdong, China. ${ }^{2}$ State Key Laboratory for Biocontrol, School of Life Sciences, Sun Yat-Sen University, Guangzhou 510060, Guangdong, China. ${ }^{3}$ Laboratory for Functional Genomics and Systems Biology, Berlin Institute for Medical Systems Biology, Berlin 13125, Germany. ${ }^{4}$ Department of Biology, Southern University of Science and Technology, Shenzhen 518055, Guangdong, China. ${ }^{5}$ Key Laboratory of Tropical Disease Control of Ministry of Education, Institute of Human Virology, Zhongshan School of Medicine, Sun Yat-Sen University, Guangzhou 510080, Guangdong, China. ${ }^{6}$ Medi-X Institute, SUSTech Academy for Advanced Interdisciplinary Studies, Southern University of Science and Technology, Shenzhen 518055, Guangdong, China. ${ }^{7}$ Present address: Laboratory of Metabolism, Center for Cancer Research, National Cancer Institute, National Institute of Health, Bethesda, MD 20892, USA. ${ }^{8}$ Present address: Division of Theoretical Systems Biology, German Cancer Research Center, Heidelberg 69120, Germany. These authors contributed equally: Jin-Yu Yang and Xiang-Yu Deng. Correspondence and requests for materials should be addressed to W.X. (email: xiewei6@mail.sysu.edu.cn)

or to S.G. (email: gaosong@sysucc.org.cn)
} 
M

odulation of translational machinery is a critical strategy for cells to overcome varied environmental assaults including viral infections ${ }^{1-4}$. Known mechanisms involve the intervention with eukaryotic translation initiation factor $4 \mathrm{~F}$ complex (eIF4F complex) or eIF2-GDP, which promotes the formation of so-called stress granules and eventually causes rapid inhibition of bulk protein synthesis ${ }^{1,5}$. Besides the regulation of translation initiation, the turnover of tRNA and rRNA has also been suggested to play an important role in translational control ${ }^{6-8}$. Stress-induced cleavage of tRNA and rRNA in the cytosol is observed in both prokaryotes and eukaryotes, which causes protein synthesis arrest and results in growth inhibition or eventually, cell death ${ }^{8-10}$. In addition, emerging evidence suggests that the cleavage products of tRNA and rRNA may act as siRNA or miRNA in promoting translation inhibition $^{11-13}$. Up to now, few tRNases involved in this process are known in high eukaryotes. These enzymes, such as human angiogenin, are normally secreted or sequestered proteins that gain access to cytosolic tRNAs upon cellular stress $8,14,15$. More RNases responsible for stress-induced tRNA/rRNA turnover are yet to be identified.

Schlafen proteins (SLFNs) were first discovered in regulating mice thymocyte development ${ }^{16}$, and then found to span across mammals with great diversity ${ }^{17}$. There are ten known or predicted SLFNs in mice which can be categorised into three subgroups by size, whereas only five SLFNs have been identified in humans, and four of them (SLFN5, SLFN11, SLFN13 and SLFN14) belong to the largest subgroup III $^{17}$ (Supplementary Fig. 1a). SLFNs were reported to be interferon (IFN)-inducible ${ }^{18-}$ 21 , and their functions have been implicated in various biological processes including cell differentiation, tumour suppression, immune response regulation and the restriction of viruses ${ }^{22}$. Specifically, human SLFN11 has been reported to bind tRNAs and restrict HIV protein synthesis possibly by means of codonbias discrimination ${ }^{23}$. However, the detailed structural properties and functional mechanisms of SLFNs still remain unclear.

All SLFNs share a specific N-terminal AAA_4 domain (Pfam04326, termed $\mathrm{N}^{\prime}$-domain in this paper) which has a very limited sequence homology to other known proteins ${ }^{17}$ and was reportedly involved in tRNA manipulation for certain members $^{23,24}$. In addition, a SWADL domain is present in the members of subgroups II and III, and the largest SLFN subgroup is characterised by a C-terminal region that is homologous to the superfamily I of RNA helicases ${ }^{25}$ (Supplementary Fig. 1a). To gain a functional insight into the Schlafen family, we determined the crystal structure of SLFN13's $\mathrm{N}^{\prime}$-domain. Investigation of the structure and subsequent functional analysis reveals that SLFN13 is a novel tRNA/rRNA-targeting RNase with potent anti-HIV activity. These results expand our vision of translational control in high eukaryotes and functional mechanisms of the Schlafen family.

\section{Results}

Overall structure of the SLFN13 N'-domain. The structure of rat (r)SLFN13 (related to human [h]SLFN13 and mouse [m]SLFN8) N '-domain containing residues $14-353$ was solved at $3.18 \AA$, where the anomalous signals of 31 selenium atoms in the asymmetric unit were used for phasing. In the calculated electron density, the main chains and most side chains were clearly traceable, enabling us to unambiguously assign them. The final model was refined to an $R_{\text {free }}$ of 0.247 (Table 1, Fig. 1a, b; Supplementary Fig. 1b). 15 vectorencoded residues fused N-terminally to SSLFN13 $_{14-353}$ are clearly discernable in the model. The U-pillow-shaped rSLFN13 ${ }_{14-353}$ is composed of an N-terminal lobe (N-lobe), a C-terminal lobe (C-lobe) and a bridging domain (BD), which together create a huge valley with a width of $\sim 23 \AA$ and a depth of $\sim 20 \AA$. rSLFN13 $14-353$

\begin{tabular}{|c|c|c|}
\hline & rSLFN13 $_{14-353}$ & rSLFN13 $14-353$ \\
\hline \multicolumn{3}{|l|}{ Data collection } \\
\hline Data set & Native & SeMet derivative \\
\hline Space group & $\mathrm{C} 2$ & $\mathrm{C} 2$ \\
\hline \multicolumn{3}{|l|}{ Cell dimensions } \\
\hline$a, b, c(\AA)$ & $172.05,134.33,77.66$ & $171.96,134.65,77.69$ \\
\hline$\alpha, \beta, \gamma\left({ }^{\circ}\right)$ & $90,105.51,90$ & $90,104.72,90$ \\
\hline Wavelength $(\AA)$ & 0.97853 & 0.97931 \\
\hline Resolution $(\AA)$ & $50.0-3.18(3.38-3.18)^{a}$ & $50.0-3.50(3.71-3.50)$ \\
\hline$R_{\mathrm{sym}}$ & $0.049(0.544)$ & $0.086(0.525)$ \\
\hline$|/ \sigma|$ & $17.08(2.05)$ & $12.00(2.81)$ \\
\hline Completeness (\%) & $99.2(98.4)$ & $99.4(99.3)$ \\
\hline Redundancy & $3.41(3.36)$ & $3.84(3.87)$ \\
\hline \multicolumn{3}{|l|}{ Refinement } \\
\hline Resolution $(\AA)$ & $35.24-3.18(3.30-3.18)$ & \\
\hline No. reflections & $28,212(2660)$ & \\
\hline$R_{\text {work }} / R_{\text {free }}$ & $0.200 / 0.247$ & \\
\hline Average $B$-factor & 108.67 & \\
\hline \multicolumn{3}{|l|}{ R.m.s. deviations } \\
\hline Bond lengths $(\AA)$ & 0.003 & \\
\hline Bond angles $\left(^{\circ}\right)$ & 0.635 & \\
\hline \multicolumn{3}{|l|}{$\begin{array}{l}\text { Ramachandran plot } \\
(\%)\end{array}$} \\
\hline Favoured & 94.48 & \\
\hline Allowed & 4.97 & \\
\hline Outliers & 0.55 & \\
\hline
\end{tabular}

a Values in parentheses are for highest-resolution shell

exhibits a pseudo-dimeric organisation: each lobe contains a fourstranded $\beta$-sheet surrounded by three $\alpha$-helices, and the bipartite $\mathrm{BD}$ derived from two separate regions of the primary sequence also bears a two-fold symmetry in topology (Fig. 1b, c; Supplementary Fig. 1c).

The $\alpha 2^{\mathrm{N}}$ and $\alpha 2^{\mathrm{C}}$ of the two lobes individually wedge into the grooves of the butterfly-like BD at both sides (Fig. 1b). N-lobe-BD association includes a hydrogen bond between Asn 54 of $a 2^{\mathrm{N}}$ and the backbone oxygen of Cys125, as well as a carboxylate- $\pi$ stacking between Glu47 of $\alpha 2^{\mathrm{N}}$ and Trp328 of $\beta 2^{\mathrm{B}}$ (Fig. 1d). Corresponding associations were found at equivalent positions of C-lobe-BD interface (Asn236-Val320 $\mathrm{O}$ and Glu229-Tyr132), although an extra salt bridge between Glu210 and Arg134 is also present (Fig. 1e). Moreover, a bulging loop of C-lobe is tightly associated with BD by a line of main chain hydrogen bonds and a CCCH-type zinc finger (Fig. 1f; Supplementary. 1d). Mutation on any of the $\mathrm{Zn}^{2+}$-coordinating residue rendered rSLFN13 1 -353 insoluble, suggesting that the zinc finger is crucial for the folding of rSLFN13. rSLFN13 ${ }_{1-353}$ constructs were eluted as monomers in the right-angle light scattering (RALS) analysis, whereas the crystallised His $_{6}$-tagged rSLFN13 $3_{14-353}$ was a dimer (Supplementary Fig. 2a). The dimerisation of $\mathrm{His}_{6}$-tagged $\mathrm{rSLFN13}_{14-353}$ is likely mediated by the artificial $\mathrm{N}$-terminus (Supplementary Fig. 2b). In agreement with this, $\mathrm{His}_{6}$-tagged rSLFN13 ${ }_{14-353}$ showed much higher resistance to the proteolytic treatment targeting $\mathrm{His}_{6}$-tag compared to $\mathrm{His}_{6}$-tagged rSLFN13 $_{1-353}$ (Supplementary Fig. 2c). In this paper, the $\mathrm{N}^{\prime}$-domain constructs of SLFN13 are collectively termed SLFN13-N unless otherwise specified. The same nomenclature is also applied to other SLFNs.

SLFN13 $\mathrm{N}^{\prime}$-domain cleaves tRNA in vitro. Close relatives of SLFN13 were reported to functionally associate with tRNA ${ }^{23,24}$, but the detailed mechanism has not been characterised yet. To understand whether and how SLFN13 is involved in tRNA 
a

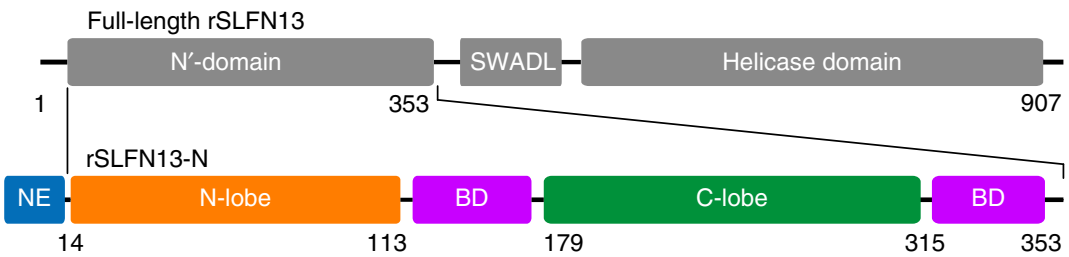

b
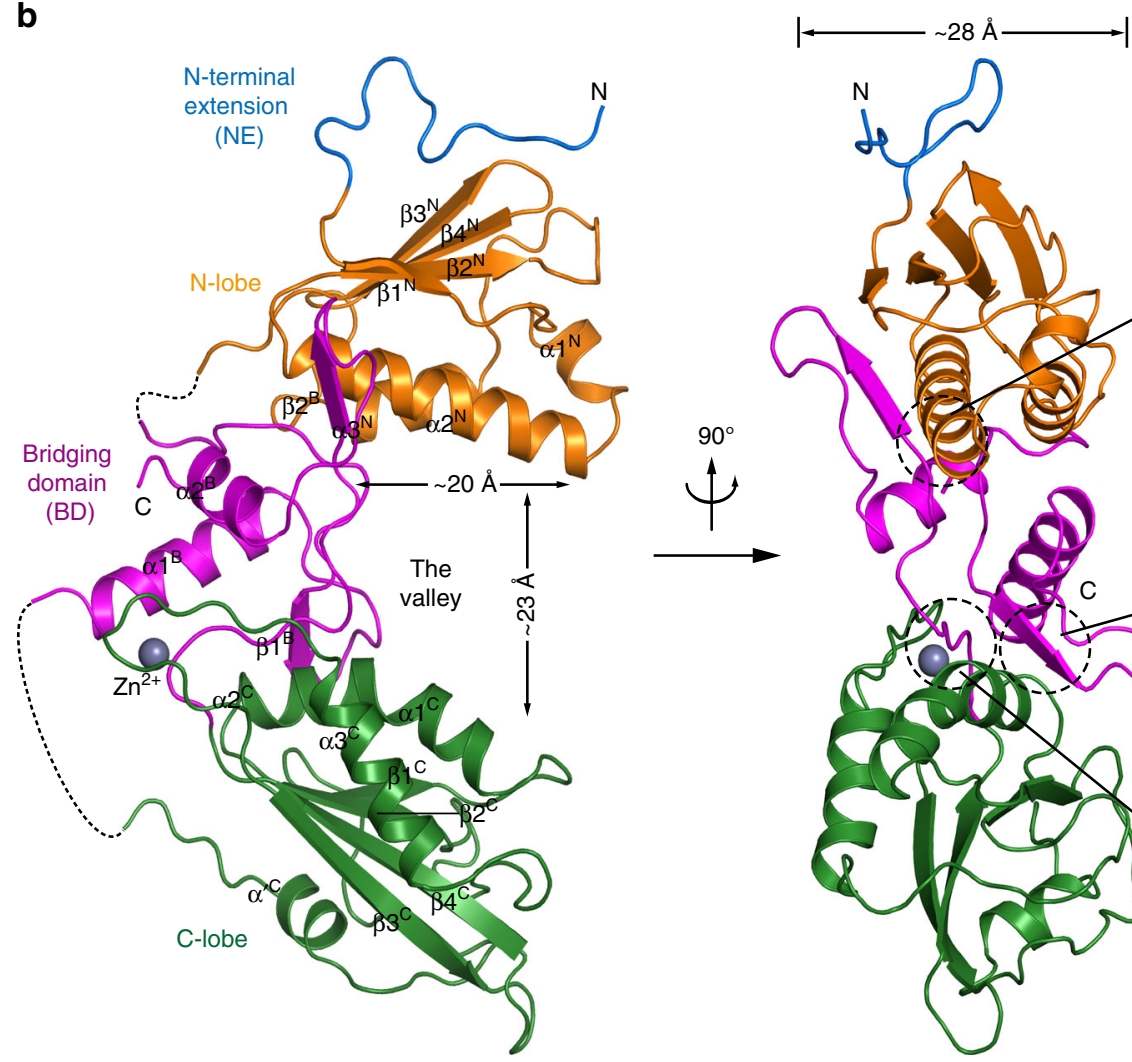

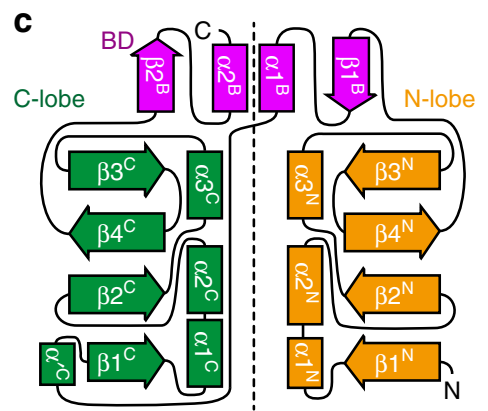

Fig. 1 Structure of rSLFN13 N'-domain. a Schematic representation showing the organisation of crystallised rSLFN13 $14-353$ based on full-length rSLFN13. NE denotes the $\mathrm{N}$-terminal extension coded by the DNA sequence from the vector; BD denotes bridging domain. Elements for rSLFN13 $14-353$ are assigned according to the structure. Borders of each element are indicated by residue numbers. $\mathbf{b}$ Structure of rSLFN13 $14-353$. Domains of rSLFN13 $14-353$ are indicated and coloured as in $\mathbf{a}$. Disordered loops are shown as dashed lines. The $\mathrm{Zn}^{2+}$ ion in the zinc finger is shown as a grey sphere. $\alpha$-helices and $\beta$-strands of each domain are specified. The size of the valley is indicated. c The topology diagram of rSLFN13 14-353. Secondary structural elements were not drawn to scale. Elements of rSLFN13 ${ }_{14-353}$ are named and coloured as in $\mathbf{a}$. A dashed line is drawn to indicate the pseudo-symmetry of rSLFN13 ${ }_{14-353}$. $\mathbf{d}$ Interactions between $\mathrm{BD}$ and $\mathrm{N}$-lobe. Side chains of involved residues are shown in the same colour as the domains they belong to. e Interactions between $\mathrm{BD}$ and $\mathrm{C}$ lobe. Note the similarity with $\mathbf{d}$. $\mathbf{f}$ Extra interactions between $\mathrm{BD}$ and $\mathrm{C}$-lobe. Note the $\mathrm{CCCH}$-type zinc finger

manipulation, we tested the binding of SLFN13-N with in vitro transcribed human tRNA Gly or tRNA ${ }^{\text {Ser }}$ (human tRNA transcripts were used in the functional assays throughout this paper, unless otherwise specified). tRNA Gly is a typical 74-nt tRNA molecule, whereas the 85 -nt tRNA $^{\text {Ser }}$ has a longer variable loop (Supplementary Table 1). In the electrophoretic mobility shift assay (EMSA), we did not observe a clear migration shift of tRNA for rSLFN13-N or hSLFN13-N. Interestingly, the tRNA seemed degraded as the concentration of both SLFN13-N increased (Supplementary Fig. 3a). Further experiments confirmed that rSLFN13-N preferably digested tRNAs over other types of nucleic acids in a protein concentration-dependent manner, and this activity also relied on $\mathrm{Mn}^{2+} / \mathrm{Mg}^{2+}$ (Fig. 2a-c; Supplementary Fig. 3b, c and Supplementary Table 2). Regarding other soluble SLFN N'-domains that could be purified in our experiments, the tRNA-cleaving feature was observed for hSLFN13-N and mSLFN8-N, but not for hSLFN5-N. Moreover, hSLFN13-N exhibited the highest cleaving efficiency and specificity among these proteins (Fig. 2d). We expanded the target tRNA types, and found that hSLFN13-N was more active than rSLFN13-N in almost all cases (Supplementary Fig. 3d, Supplementary Table 1). In addition, purified full-length mSLFN1 lacks nucleolytic activity on given tRNAs (Supplementary Fig. 3e). These results exemplify the diversity of SLFNs in tRNA manipulation.

The processing pattern of SLFN13-N for tRNAs. hSLFN13, mSLFN8 and rSLFN13 share a common cleavage product (specified as primary product, Fig. 2d), and this product appeared for all tRNA targets (Supplementary Fig. 3d), suggesting that the cleavage by SLFN13 takes place first on a specific site of tRNAs. To figure out the exact cleavage site, we generated tRNA ${ }^{\text {Ser }}$ molecules with differentially modified termini and tested them in the digestion assay. Extension of the $5^{\prime}$ end by a guanosine ( $5^{\prime}-\mathrm{G}$ $\mathrm{tRNA}^{\text {Ser }}$, V1) led to larger product bands, whereas shortening of 
a

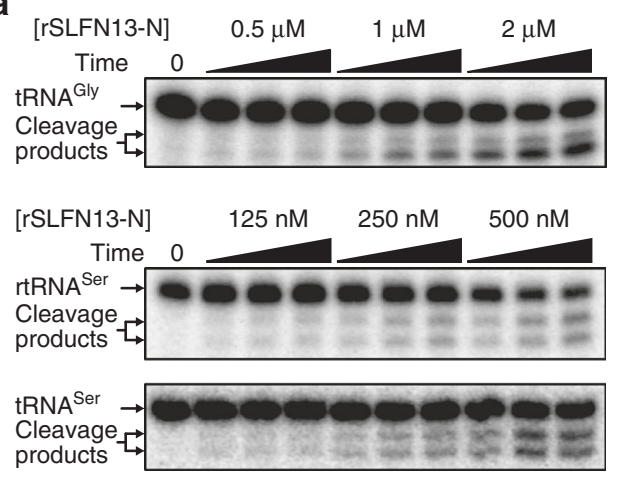

c

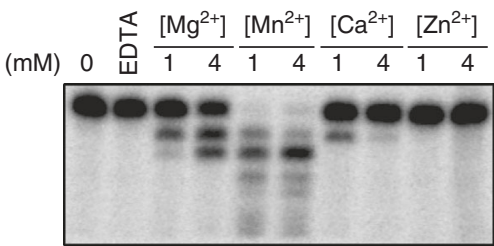

d

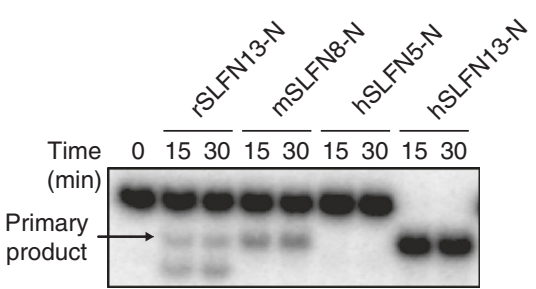

f

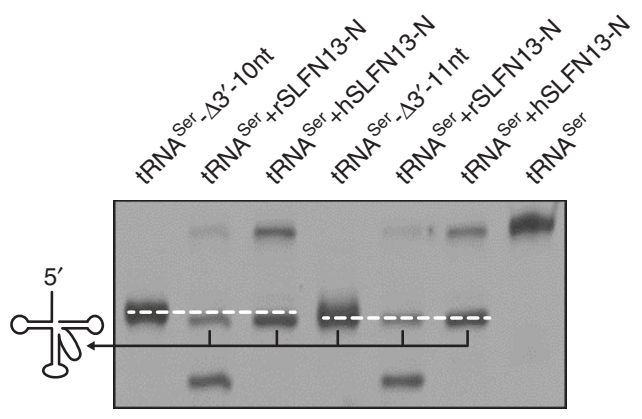

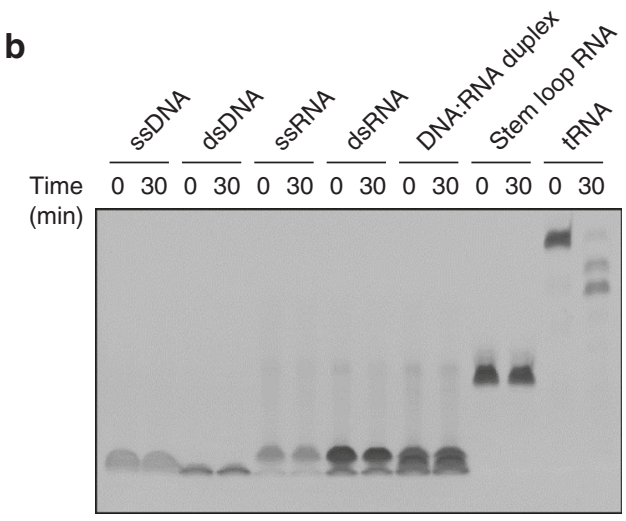

e

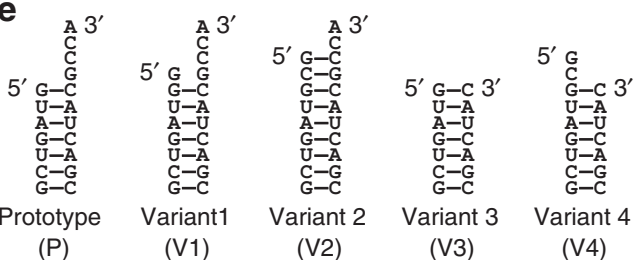

(P)

(V2)

(V3)

(V4)

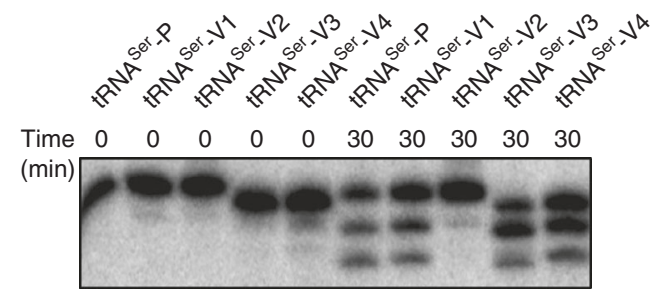

g

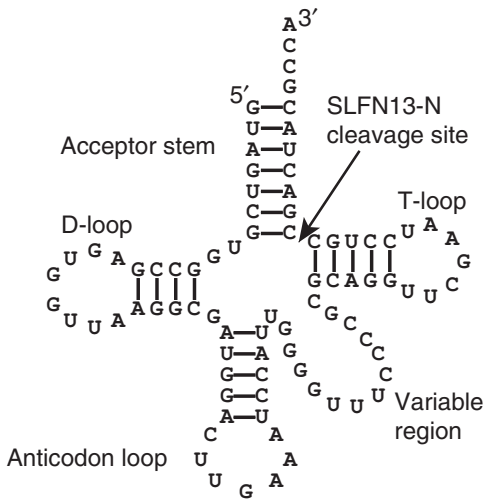

Fig. 2 Endoribonuclease activity of SLFN13-N. a Dose-dependent and time-dependent tRNA digestion by rSLFN13-N in the presence of $\mathrm{Mg}^{2+}$. rSLFN131-353 of indicated concentrations were incubated with [ $\alpha^{-32}$ P]-labelled human tRNA ${ }^{\text {Gly }}$, human tRNA ${ }^{\text {Ser }}$ or rat tRNA ${ }^{\text {Ser }}$ ( $r$ tRNA ${ }^{\text {Ser}}$ ) for 5,15 and 30 min. b rSLFN13-N preferably cleaves tRNA. ssDNA/ssRNA single-stranded DNA/RNA, dsDNA/dsRNA double-stranded DNA/RNA. c Analysis of divalent cations as cofactor of rSLFN13-N for tRNA digestion. $500 \mathrm{nM}$ rSLFN13 $1-353$ was incubated with [ $\left.\alpha^{-32}{ }^{32}\right]$-labelled tRNA ${ }^{\text {Ser }}$ for 30 min. Four different divalent cations were individually tested. $\mathbf{d}$ Cleavage assay for different SLFN-N proteins. $250 \mathrm{nM} \mathrm{rSLFN13} 1_{-353}$, mSLFN8 ${ }_{1-359}$, hSLFN131-355 or hSLFN5 12-334 $_{12}$ was individually incubated with $\left[\alpha^{-32}{ }^{32}\right]$-labelled tRNA ${ }^{\text {Ser }}$. Note that hSLFN13-N shows highest nucleolytic efficiency over human tRNA ${ }^{\text {Ser }}$. e Cleavage assay for rSLFN13-N on acceptor stem variants. Modifications of the variants are specified. $500 \mathrm{nM}$ rSLFN13 $1-353$ was used in each reaction. $\mathbf{f}$ tRNA cleavage pattern of SLFN13$\mathrm{N}$. The primary cleavage products from tRNA ${ }^{\mathrm{Ser}}$ by rSLFN131-353 and hSLFN131-355 were compared with in vitro transcribed tRNA $A^{\text {Ser }}$ that lacks 3'-terminal $10 \mathrm{nt}\left(\mathrm{tRNA}{ }^{\mathrm{Ser}}-3^{\prime}-\Delta 10 \mathrm{nt}\right)$ or $11 \mathrm{nt}\left(\mathrm{tRNA}{ }^{\mathrm{Ser}}-3^{\prime}-\Delta 11 \mathrm{nt}\right)$, and portrayed to the left. The migration of two truncated tRNA ${ }^{\mathrm{Ser}}$ markers is indicated by dashes lines. $\mathbf{g}$ Proposed primary cleavage site of SLFN13-N. The sequence of human tRNA ${ }^{\mathrm{Ser}}$ is used here as representative

the $3^{\prime}$ end (tRNA ${ }^{\text {Ser }}-3^{\prime}-\triangle$ GCCA, V3) had no effect (Fig. 2e). This means the original $5^{\prime}$ end of tRNA ${ }^{\text {Ser }}$ was preserved in the primary product, and the cleavage site is close to the $3^{\prime}$ end. When two extra bases (GC) were added to the $5^{\prime}$ end of the tRNA ${ }^{\text {Ser }}\left(5^{\prime}\right.$ GC-tRNA $\left.{ }^{\text {Ser }}, \mathrm{V} 2\right)$ which extended the acceptor stem from 7 to 9 bp, the tRNA became resistant to SLFN13. Once the GCCA motif at the $3^{\prime}$ end was removed from this tRNA molecule (5'-GCtRNA $^{\text {Ser }}-3^{\prime}-\triangle \mathrm{GCCA}, \mathrm{V} 4$ ), it became cleavable again (Fig. 2e). These results indicate that the length of the paired bases of the tRNA acceptor stem is important for the selectivity of SLFN13. By comparing the migration rate of SLFN13-digested tRNA ${ }^{\mathrm{Ser}}$ with designated markers in electrophoresis assay, we found that the 
a

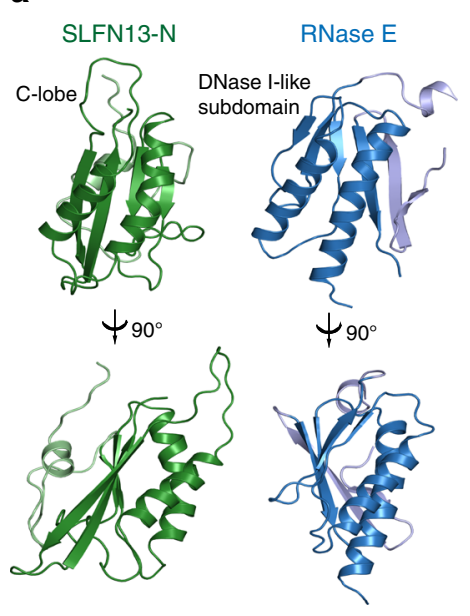

b

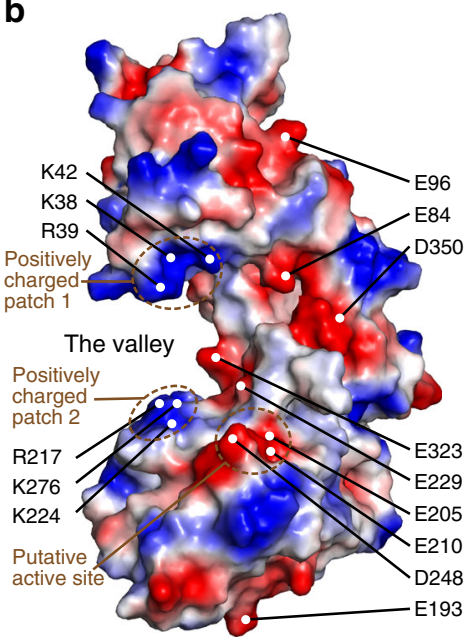

C

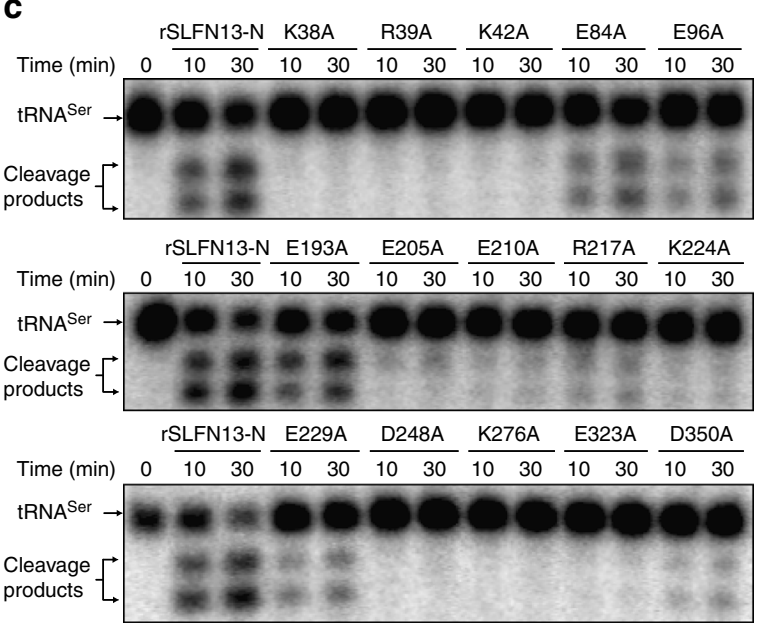

d

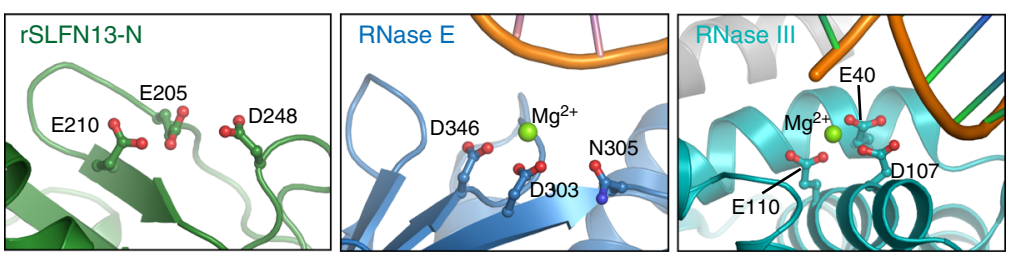

$\mathbf{f}$

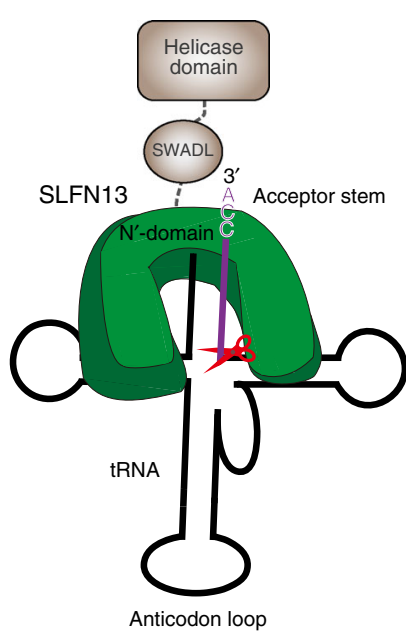

g

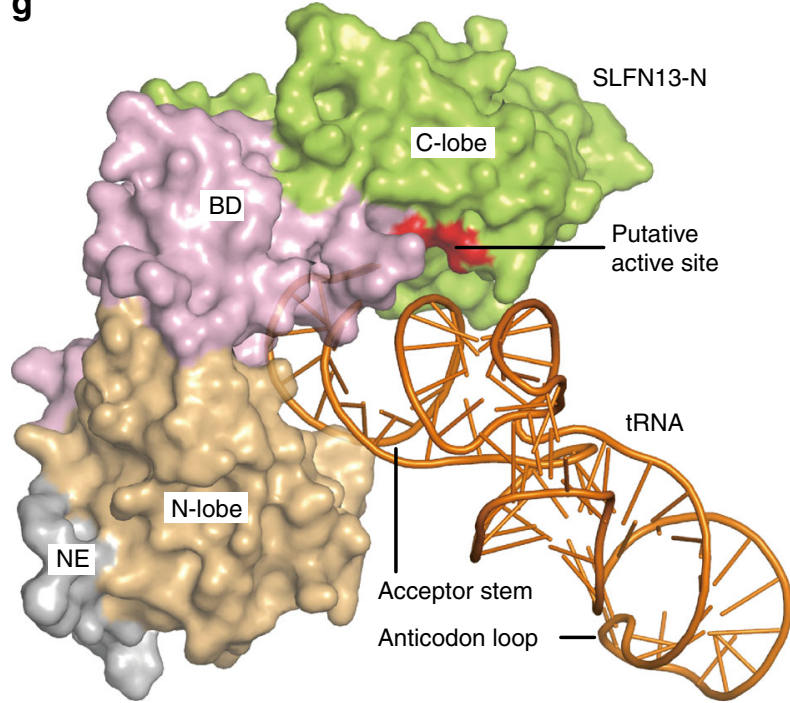

e

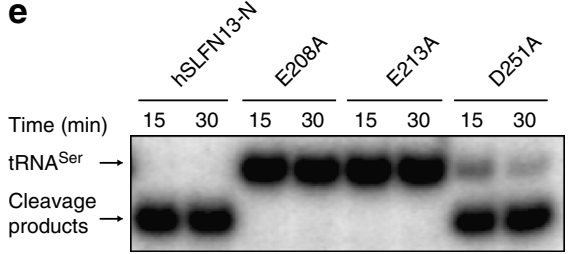

h

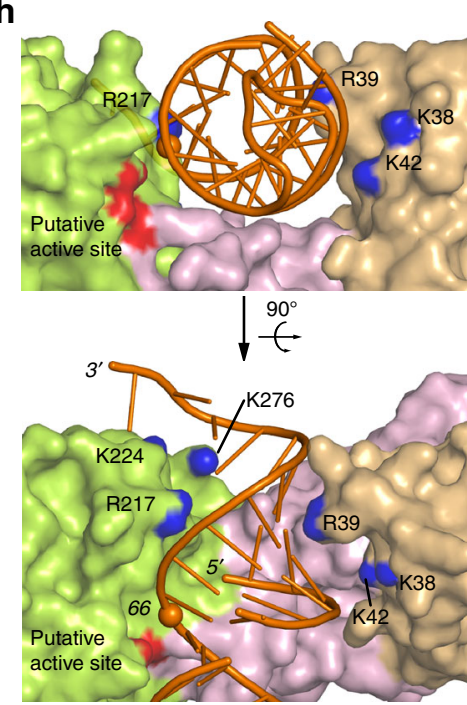

Fig. 3 Putative catalytic site and cleavage model of SLFN13-N. a Structural comparison between the C-lobe of rSLFN13 $14-353$ and the DNase I subdomain of RNase E (PDB ID 2BX2). For RNase E, the portion that is structurally aligned to rSLFN13 $14-353$ C-lobe is shown in blue, and the rest part in light blue. $\mathbf{b}$ The electrostatic surface potentials of rSLFN13 $14-353$, coloured from red (negative) to blue (positive). Two negatively charged patches and the conserved positively charged area are indicated. Positions of the residues tested in c are specified. c tRNA cleavage assay for rSLFN13 $1-353$ mutants of conserved charged residues in the presence of $\mathrm{Mg}^{2+}$. For each sample, $500 \mathrm{nM}$ protein was used. $\mathbf{d}$ Comparison between the putative nucleolytic active site of rSLFN13-N and the actives sites of RNase E (2BX2) and RNase III (2EZ6). Note the similar tripod architecture of the three residues for each protein, which are shown as ball-and-stick models. e Cleavage assay of hSLFN13 $1-355$ mutants at the putative active site. E208A, E213A and D251A of hSLFN13-N correspond to E205, E210 and D248 of rSLFN13-N, respectively. f Schematic drawing of SLFN13 cleaving a tRNA. The U-pillow-shaped SLFN13 N'-domain embraces the acceptor stem of the tRNA. The 3' tail that is to be cleaved off the tRNA by SLFN13-N is coloured purple. $\mathbf{g}$ Structural model of SLFN13-N manipulating tRNA. rSLFN13 $14-353$ is shown as surface representations and the subdomains are colour-specified. The putative active site is coloured red. The coordinate of tRNA Gly (5E6M, excerpted) is used and coloured orange. SLFN13-N clamps the acceptor stem of tRNA and may have no contact with other parts of the tRNA. $\mathbf{h}$ Close view of SLFN13-N accommodating the acceptor stem. The surface positively charged residues of rSLFN1314-353 tested in $\mathbf{c}$ are coloured blue. Part of the tRNA is removed for clarity. The $5^{\prime}$ and $3^{\prime}$ ends of the tRNA are indicated. The phosphate atom of the 66 th nucleotide of tRNA is shown as a sphere 
a

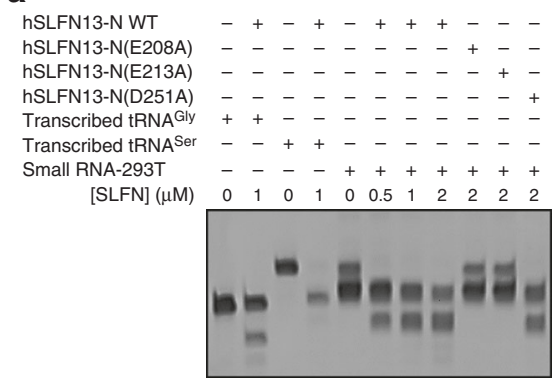

d

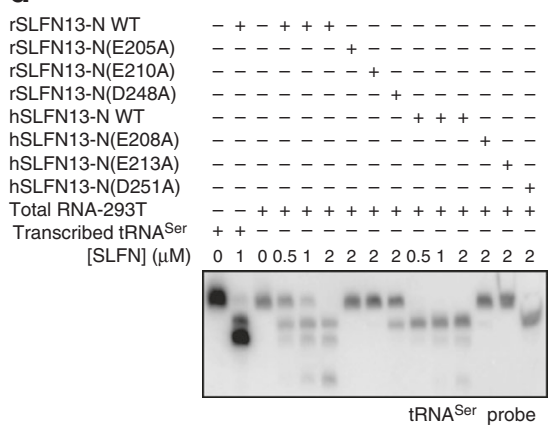

g

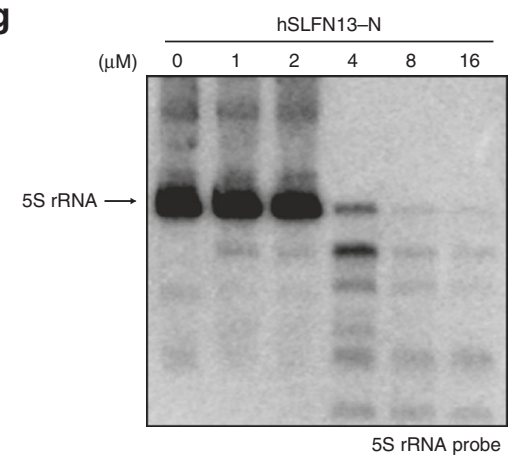

b

ISLFN13-N WT

rSLFN13-N(E205A) - $-\ldots+\ldots+\ldots$

ISLFN13-N(E210A) - - - - - - -

rSLFN13-N(D248A)

Transcribed tRNA Gly

Transcribed tRNA ${ }^{\text {Se }}$

[SLFN] $(\mu \mathrm{M})$

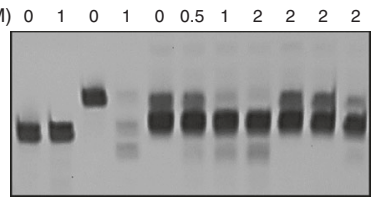

e

ISLFN13-N WT

rSLFN13-N(E205A) - -+-+------

rSLFN13-N(E210A) - - - - +

rSLFN13-N(D248A) $\ldots \ldots \ldots+\ldots$

hSLFN13-N WT $\ldots \ldots \ldots$

hSLFN13-N(E208A) - $\ldots \ldots$

hSLFN13-N(E213A) _ - . - . . + +

hSLFN13-N(D251A) $\ldots \ldots \ldots \ldots+\ldots$

Total RNA-293T +++++++++++++

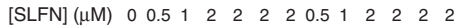

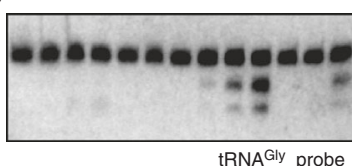

h

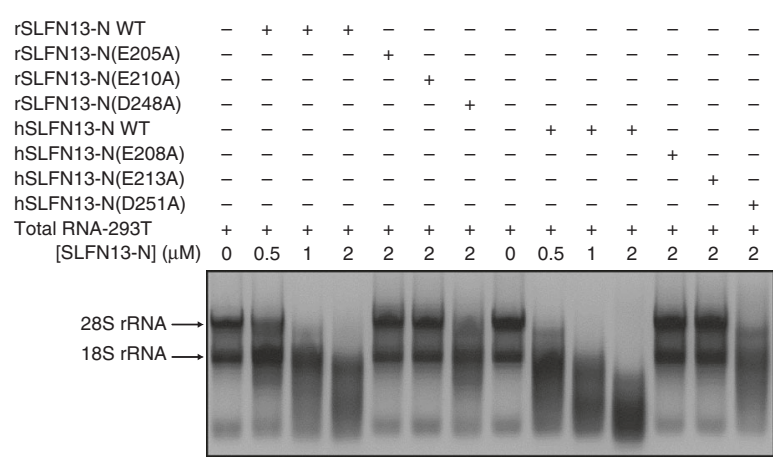

Fig. 4 SLFN13-N cleaves native tRNA and rRNA. a, b Cleavage assay of hSLFN13-N (a) and rSLFN13-N (b) on native tRNAs in the presence of Mg $2+$. Small RNA-293T denotes total small RNA extracted from 293T cells that is mainly constituted of tRNAs. tRNA ${ }^{\text {Ser }}$ and tRNA ${ }^{\text {Gly }}$ were used as controls to indicate molecular weight. c Cleavage assay of different SLFN-Ns on native tRNAs. d-f Cleavage assay on specific native tRNAs. The substrates and products were identified by Northern blot using $\left[\gamma^{-}{ }^{32} \mathrm{P}\right]$-labelled probes targeting tRNA ${ }^{\text {Ser }}(\mathbf{d})$, tRNA ${ }^{\text {Gly }}$ (e) or tRNA ${ }^{\text {Lys }}$ (f). $\mathbf{g}$ Cleavage assay for hSLFN13-N on 5S rRNA. $5 \mathrm{~S}$ rRNA and products were identified by Northern blot. $\mathbf{h}$ Cleavage assay on native rRNAs. $4 \mu \mathrm{g}$ total RNA extracted from $293 \mathrm{~T}$ cells was used for each reaction, which mainly contains rRNAs

primary cleavage takes place on the acceptor stem of the substrate tRNA and most likely removes $11 \mathrm{nt}$ at the $3^{\prime}$-terminus (Fig. 2f, g). For some tRNAs, a 'secondary' cleavage product could be observed, which seems to be derived from further digestion by SLFN13-N as it never appeared alone (Fig. 2a-e; Supplementary Fig. 3b-d). According to the size and migration feature of this product, we infer that the secondary cleavage takes place on the T-loop, which dissevers another 11 or $12 \mathrm{nt}$ from the primary product (Supplementary Fig. 3f).

To further investigate the selectivity of SLFN13-N on tRNAs, we assayed several types of tRNAs from various species for SLFN13-N cleavage. Most of the tRNAs were able to be digested by hSLFN13-N, whereas rSLFN13-N seemed more active to those tRNAs with longer variation loops (Supplementary Fig. $4 \mathrm{a}-\mathrm{d}$ and Supplementary Table 1). Interestingly, both hSLFN13-N and rSLFN13-N lacked the endoribonuclease activity on $\mathrm{tRNA}^{\mathrm{Sec}}$ that possesses a 9-bp acceptor stem (Supplementary Fig. 4c, d). This result supports our finding that the length of acceptor stem is critical for the selectivity of SLFN13-N (Fig. 2e).
C
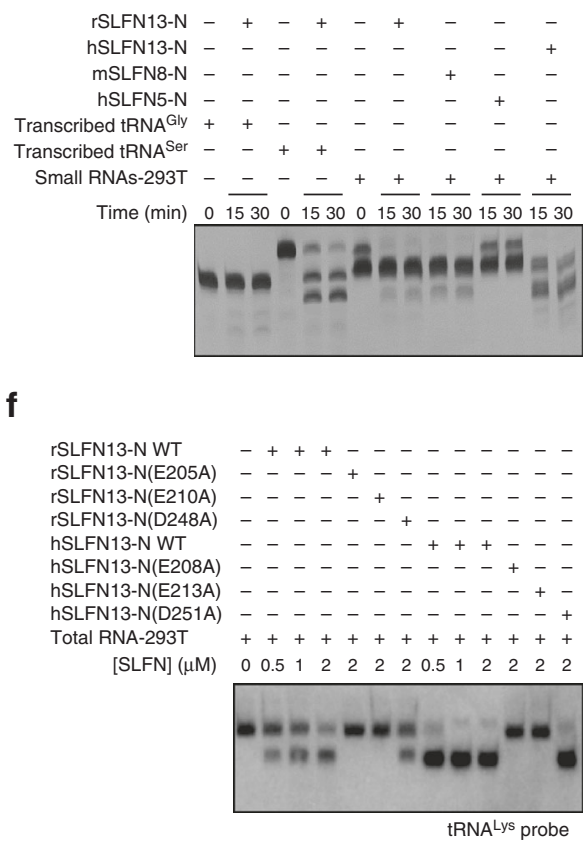
a

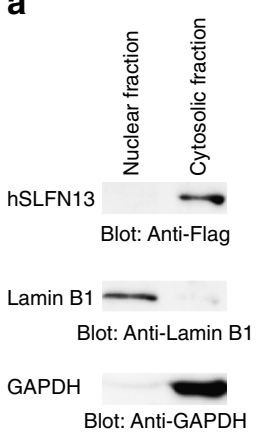

b

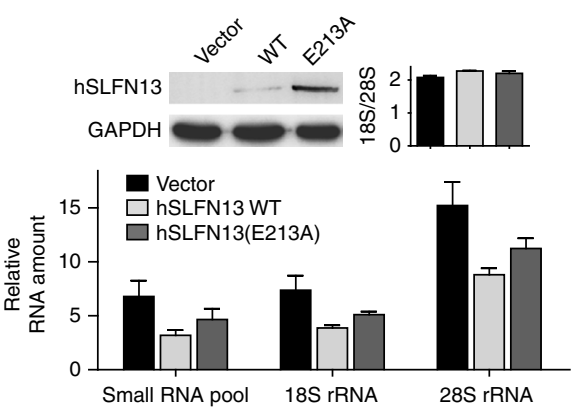

d

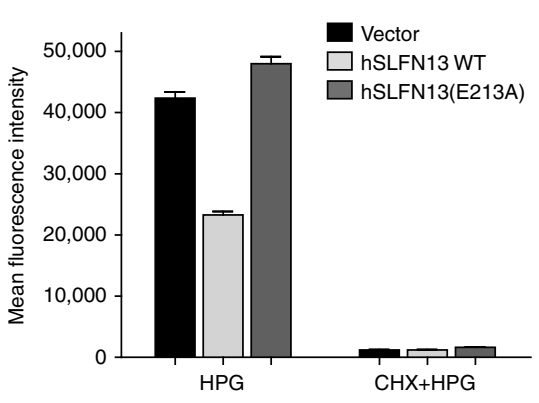

e

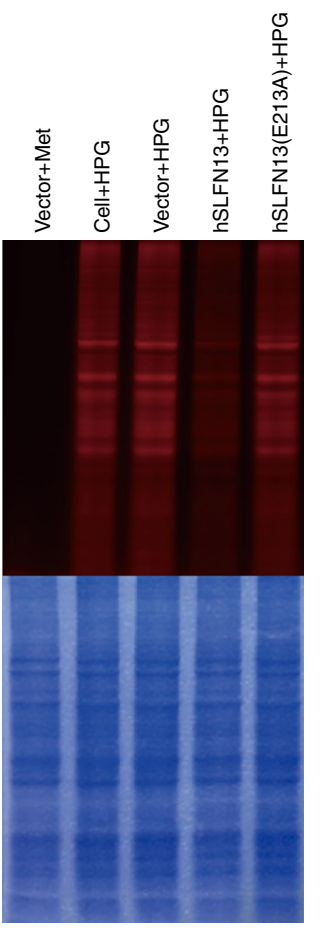

Homopropargylglycine (HPG) Azidohomoalanine-Cy5 (AHA-Cy5)

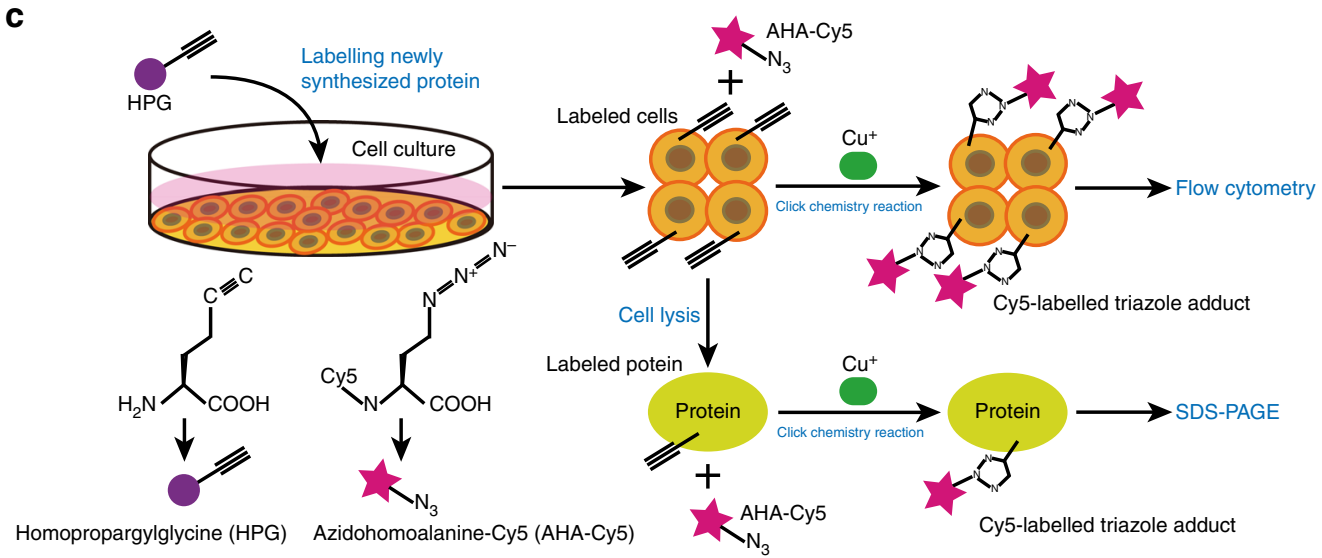

Fig. 5 hSLFN13 disrupts translational machinery. a Subcellular localisation of hSLFN13. b hSLFN13 restricts tRNAs and rRNAs in cells. The small RNA pool is mainly constituted of tRNAs and 5S/5.8S rRNA (See Supplementary Fig. 8f). Expression of hSLFN13 WT and hSLFN13(E213A) was confirmed by Western blot. Note the prominent restriction of cellular RNAs caused by relatively low level of overexpressed hSLFN13. Error bar indicates s.d. $(n=3)$. c The experimental process of the FUNCAT-click chemistry assay. d FUNCAT assay and subsequent flow cytometry examination for Cy5 fluorescence showing that hSLFN13 inhibits protein synthesis in living cells. e hSLFN13 inhibits protein synthesis. Upper: fluorescent image of the SDS-PAGE gel showing the newly synthesised proteins labelled by FUNCAT assay. Lower: same gel stained with Coomassie Blue showing the loading consistency

The DNase I-like subdomain is a component of the RNase E catalytic domain that forms a homotetramer ${ }^{33}$. Within this tetramer, the DNase I subdomain dimerises and cleaves two singlestranded RNA at both sides of the dimer (Supplementary Fig. 5e). Compared with RNase E, native rSLFN13-N is a monomer in solution (Supplementary Fig. 2a), and has distinct overall architecture and substrate preference. Thus, although bearing putative evolutional traces from the bacterial RNases, SLFN13-N represents a novel class of endoribonuclease.

Critical regions of SLFN13-N for tRNA cleavage. Surface electrostatic potential analysis of rSLFN13-N revealed two positively charged patches inside the valley, whose width is exactly suitable for accommodating base-paired RNA (Fig. 3b). Substitution of conserved charged residues (Lys38, Arg39, Lys42, Arg217, Lys224, Lys276) to alanine resulted in reduced nucleolytic activity, suggesting that rSLFN13-N may embrace base-paired RNA within its valley (Fig. 3c). Apart from the positively charged patches, a negatively charged area formed by conserved Glu205, Glu210 and Asp248 of the C-lobe is also observed at the rim of the valley (Fig. 3b; Supplementary Figs 6 and 7a, b). The threecarboxylate triad is reminiscent of the catalytic residues of bacterial endoribonucleases involved in RNA processing, such as RNase E and RNase III, which coordinate a hydrated $\mathrm{Mg}^{2+}$, possibly for the nucleophilic attack on the scissile phosphate in the RNA backbone ${ }^{33,34}$ (Fig. 3d). Mutating any of the three residues diminished the nucleolytic activity of rSLFN13-N and hSLFN13-N (Fig. 3c, e). Even at elevated enzyme concentration, E205A and E210A mutants were absolutely inactive, which differed from other single-point mutants (D34A, E47A, E84A, E96A, E146A, D193A, E221A, E229A, E249A, D259A, E323A, $\mathrm{D} 350 \mathrm{~A})$ regarding exposed and relatively conserved aspartates or glutamates (Fig. 3c, e; Supplementary Fig. 7c-e). This result demonstrates, that Glu205 and Glu210 are pivotal residues for the endonucleolytic activity of SLFN13-N. In addition, this plausible active site appears only in the C-lobe but not in the N-lobe (Supplementary Fig. 6), suggesting that SLFN13 cleaves only on one site of base-paired RNA. This is in accordance with the results of tRNA cleavage assays (Fig. 2e).

Mechanistic model of tRNA cleavage by SLFN13-N. Based on the biochemical analysis, we propose that SLFN13-N clamps the tRNA acceptor stem and cleaves in a site-specific and sequenceindependent manner, which is likely controlled by the interplay between the inner shape of the valley and the tertiary structure of the tRNAs (Fig. 3f). To illustrate this special mechanism, we generated a structural model of SLFN13-N-tRNA complex (Fig. $3 \mathrm{~g}$ ). In this model, the acceptor stem of the tRNA is perfectly docked in the valley of SLFN13-N, where the protruding positively charged residues support the tRNA backbone, and putative active site of SLFN13-N is right next to the predicted cleavage site between the 65th and 66th nucleotide of tRNA. The $5^{\prime}$ end of tRNA is tightly packed in the valley, leaving insufficient space for an extra base (Fig. 3h). This may explain why SLFN13-N does not cleave the V2-tRNA ${ }^{\text {Ser }}$ that has a 9-bp acceptor arm (Fig. 2e). This reaction would cause the loss of the CCA tail, hence the amino-acylation of tRNA molecules. 

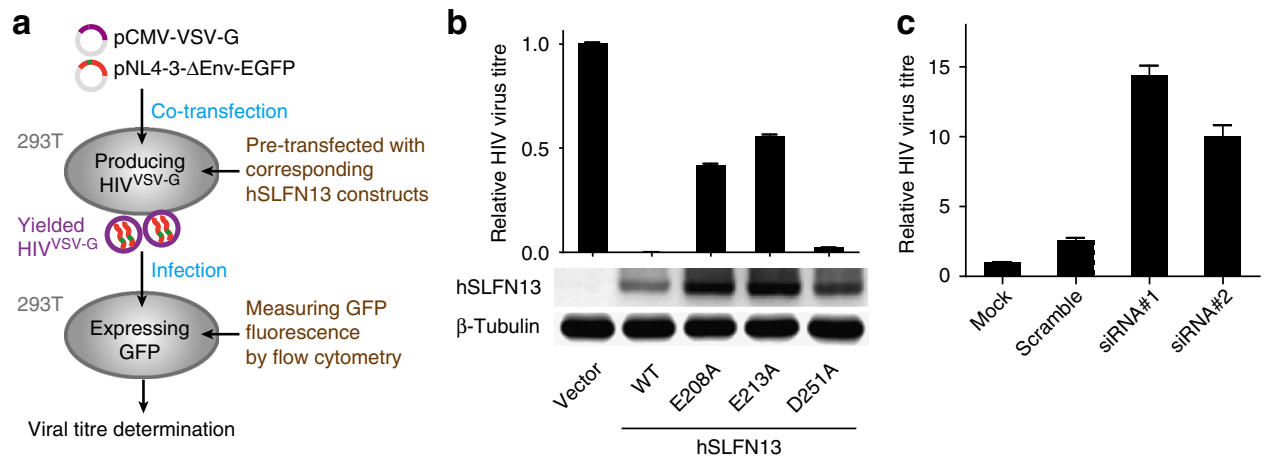

Viral titre determination

d
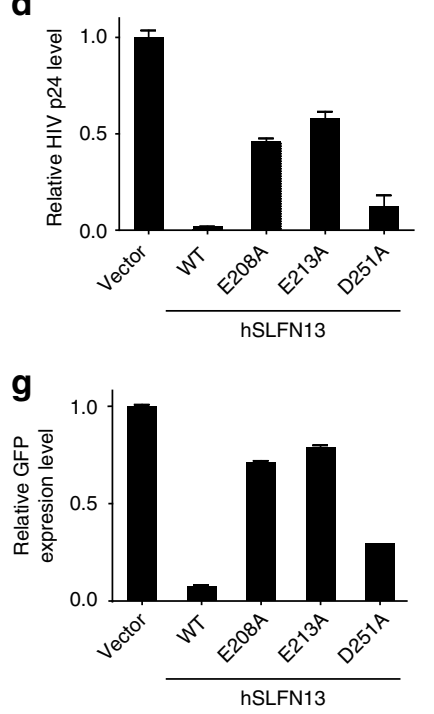

e

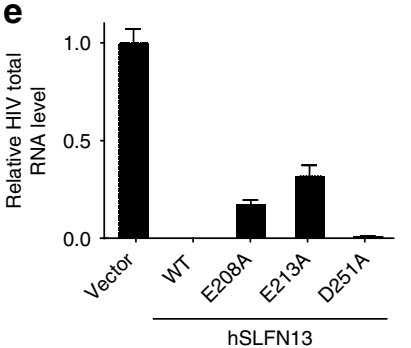

$\mathbf{h}$

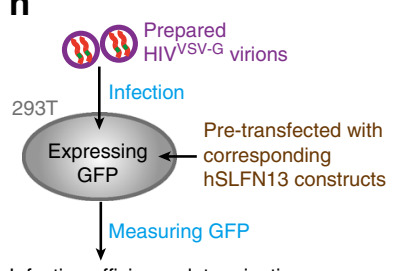

Infection efficiency determination
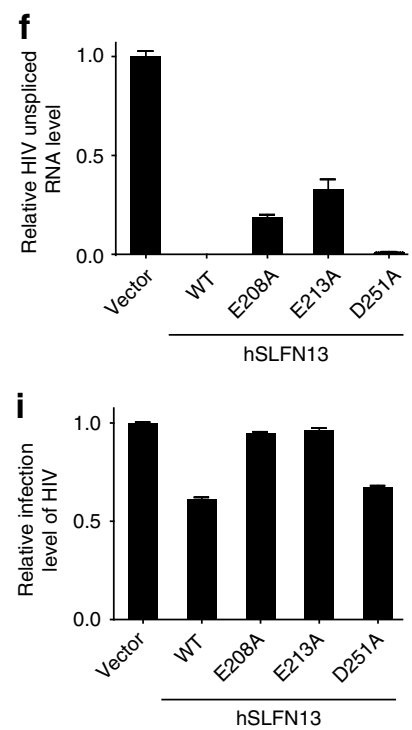

j

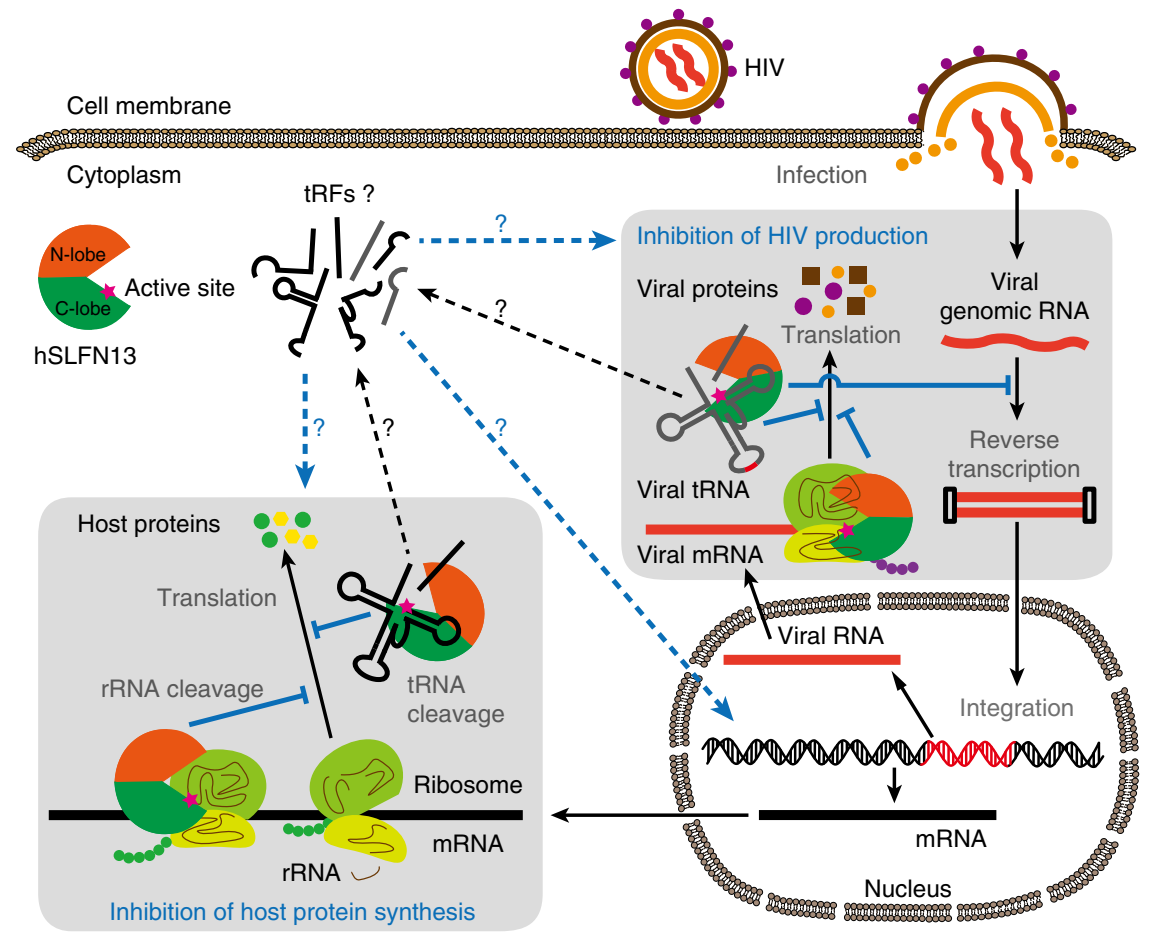

Fig. 6 hSLFN13 restricts HIV production. a The experimental process of titre assay for VSV-G pseudotyped HIV (HIVVSV-G). b Viral production was assayed by titrated infection and the GFP level. Expression of hSLFN13 WT and mutants was confirmed by Western blot. Error bar indicates s.d. ( $n=3$ ). $\mathbf{c}$ Knock-down of endogenous SLFN13 increased viral titre of 293 cells. d Viral particle content in supernatants was analysed by p24 ELISA. e, $\mathbf{f}$ Extracellular vRNA concentration was determined by qPCR for total vRNA (e) and unspliced vRNA (f). $\mathbf{g}$ hSLFN13 suppressed GFP production from the viral plasmids. $\mathbf{h}$ The experimental process of the viral infection assay. i Examination for the infection efficiency of HIVVSV-G in cells pre-transfected with hSLFN13 WT or mutants. j Proposed protein synthesis inhibition and anti-HIV mechanism of hSLFN13 
SLFN13 restricts cellular tRNA and rRNA. To understand whether SLFN13-N is able to digest natural tRNAs carrying various post-transcriptional modifications, total tRNAs isolated from HEK-293T (293T) cells or HeLa cells were applied to cleavage assays. When incubated with hSLFN13-N, mSLFN8-N or rSLFN13-N, the total tRNA showed a degradation pattern similar to the in vitro experiments in a protein concentrationdependent manner (Figs 2a, 4a-c; Supplementary Fig. 8a-c). Northern blot assays confirmed that several types of tRNA were susceptible to hSLFN13-N and rSLFN13-N digestion (Fig. 4d-f; Supplementary Table 3). In addition, SLFN13-N was able to digest the 5S, 18S and 28S rRNA extracted from 293T cells or HeLa cells (Fig. 4g, h; Supplementary Fig. 8d). We surmise, that SLFN13-N cuts rRNAs at base-paired regions that are topologically similar to the acceptor stem of tRNAs.

Next, we investigated hSLFN13 in vivo. Although several other Schlafen family members of subgroup III were reported to carry a nuclear localisation signal (NLS) ${ }^{22}$, hSLFN13 does not seem to have any NLS (Supplementary Figs 1a and 6). Overexpressed hSLFN13 showed a preference for cytoplasmic distribution in 293T cells and HeLa cells, therefore, it should be accessible to mature tRNAs and ribosomes (Fig. 5a; Supplementary Fig. 8e). Indeed, the tRNA and rRNA levels in hSLFN13-expressing cells were markedly reduced, which is apparently associated with its nucleolytic activity (Fig. 5b; Supplementary Fig. 8f). We then investigated the effect of hSLFN13 on protein synthesis based on fluorescent noncanonical amino acid labelling (FUNCAT). Newly synthesised proteins were labelled using homopropargylglycine (HPG) and detected via click chemistry (Fig. 5c). In hSLFN13-transfected $293 \mathrm{~T}$ cells, the amount of newly synthesised proteins was greatly diminished (Fig. 5d, e). These results prove that hSFLN13 can disrupt translational machinery and retard protein synthesis by restricting cytoplasmic tRNA and rRNA.

SLFN13 restricts HIV in a nucleolytic activity-dependent manner. Several subgroup III SLFNs are suggested to restrict different viruses ${ }^{21,23}$. While hSLFN13 was reported to moderately inhibit influenza virus, it is intriguing as to whether SLFN13 also inhibits other types of viruses. Expression of SLFN13 was not inducible by IFNa $/ \beta / \gamma$ treatment in $293 \mathrm{~T}$ cells, but could be stimulated by the infection of VSV-G pseudotyped HIV-1 (HIV VSVG) (Supplementary Fig. 9a, b). To confirm whether SLFN13 is capable to restrict HIV, we transfected 293T cells with pNL4-3$\Delta$ Env-EGFP (encoding essential HIV-1 proteins but the coding sequence for HIV-1 Envelope was partially replaced by the cDNA of EGFP) and pCMV-VSV-G (encoding the vesicular stomatitis virus G glycoprotein, or VSV-G that serves as a surrogate viral envelope protein), and measured the titre of VSV-G pseudotyped HIV-1 (HIV VSV-G) produced from these cells in the presence of hSLFN13/hSLFN13-N constructs (Fig. 6a). After being transfected with wild-type (WT) hSLFN13, these cells failed to yield $\mathrm{HIV}^{\mathrm{VSV}-\mathrm{G}}$ titre, indicating the restriction of HIV (Fig. $6 \mathrm{~b}$ ). In agreement with this, knock-down of SLFN13 dramatically weakened HIV resistance of 293 cells (Fig. 6c; Supplementary Fig. 9c, d). Apart from HIV, we also tested antiviral activity of SLFN13 on viruses belonging to Herpesviridae and Flaviviridae. hSLFN13 failed to confer efficient resistance against herpes simplex virus (HSV), and only moderately restricted Zika virus (Supplementary Fig. 9e, f).

The nucleolytic-deficient mutations E208A and E213A dramatically decreased the anti-HIV activity of hSLFN13, while the less destructive mutant D251A remained highly active (Fig. 6b). This anti-HIV tendency of hSLFN13 mutants is congruent with their behaviours in the tRNA cleavage assays
(Figs 3e, 4a-c; Supplementary Fig. 7d), manifesting the crucial role of hSLFN13's nucleolytic activity in HIV restriction. Moreover, hSLFN13-N also efficiently reduced viral titre, whereas hSLFN13-N(E208A) and hSLFN13-N(E213A) showed much weaker anti-HIV activity compared to their full-length counterparts (Supplementary Fig. 9g), suggesting that the SWADL domain and/or helicase domain may also contribute to the anti-HIV activity of hSLFN13.

SLFN13 blocks synthesis of viral proteins. To discern whether hSLFN13 reduced the number or the viability of the released virus, we measured viral protein and viral RNA (vRNA) levels in the supernatant of hSLFN13/hSLFN13-N-transfected, HIV VSV-G producing cells. Extracellular concentration pattern of p24 capsid, total vRNA or unspliced vRNA was consistent with the titre results, suggesting that hSLFN13 diminished the number of released viral particles (Fig. 6d-f; Supplementary Fig. 9h-j and Supplementary Table 4). Moreover, hSLFN13/hSLFN13-N suppressed the fluorescence from 293T cells transfected with viral vector pNL4-3- $\Delta$ Env-EGFP (Fig. 6g; Supplementary Fig. 9k), which was strong evidence for the disrupted synthesis of viral proteins. To clarify whether this observation resulted solely from overall protein synthesis arrest caused by hSLFN13 or hSLFN13$\mathrm{N}$, we compared the fluorescence of various EGFP-containing plasmids co-transfected with hSLFN13/hSLFN13-N in 293T cells. Plasmids with HIV promoter were obviously more susceptible than the control plasmid (Supplementary Fig. 9l, m), illustrating the preference of hSLFN13 over HIV-related protein synthesis. This preference is possibly resulted from the cleavage by SLFN13 on the HIV-derived sequences of the transcripts, as they contain stem loops which structurally resemble the SLFN13-targeting regions of tRNAs (Fig. $2 \mathrm{~g}$ ). In addition, we also checked whether hSLFN13 hinders HIV VSV-G infection (Fig. 6i). Although 293T cells pre-transfected with hSLFN13/hSLFN13-N showed a decreased GFP level (Fig. 6i; Supplementary Fig. 9n), this result may be attributable to the restriction of global protein synthesis. Thus, it is currently tentative to conclude SLFN13 affects the infection of HIV.

\section{Discussion}

Our study has revealed SLFN13 as a novel type of eukaryotic tRNA/rRNA endoribonuclease with several features. First, its unique pseudo-dimeric U-pillow-shaped architecture allows for the docking of base-paired RNA. Second, SLFN13 seems to lack the discrimination over tRNA types but relies on the recognition of the secondary or tertiary structure. Third, according to the effects of overexpressed SLFN13 in translational control of cell lines, it is possible that upon certain cellular stresses, induced SLFN13 inhibits protein synthesis by directly digesting cytoplasmic tRNA and rRNA, so as to realise a fast stress response. Fourth, SLFN13 is so far the only eukaryotic endoribonuclease that has been proven to preferably cleave at the acceptor stem of mature tRNAs. Many other cytosolic tRNA processors target the anti-codon loop, such as stress-induced mammalian angiogenin ${ }^{15,35}$ and yeast Rnylp $^{14}$, as well as E. coli PrrC and colicin D/E5 involved in the bacterial defence system ${ }^{9,36-38}$. In bacteria, the secreted bacterial toxin CdiA family involved in contact-dependent growth inhibition was also reported to cut the acceptor stem of certain tRNAs ${ }^{39}$. However, unlike SLFN13, the in vitro nucleolytic activity of CdiA family proteins is dependent on the association with elongation factor $\mathrm{Tu}(\mathrm{EF}-\mathrm{Tu})$ and the presence of GTP ${ }^{40}$. According to these properties, we propose a novel nomenclature for SLFN13: RNase S13. 
The nucleolytic activity of hSLFN13 is crucial for its anti-HIV potency. The cleavage of cytoplasmic tRNA and rRNA by hSLFN13 may cause global translation inhibition that suppresses both viral replication and cell viability to confine viruses (Fig. 6j). A similar mechanism was discovered for the suicidal tRNase PrrC in E. coli, which cleaves its own tRNA ${ }^{\mathrm{Lys}}$ in response to bacteriophage T4 infection ${ }^{36}$. This may explain a recent observation that marmoset SLFN11 blocks synthesis of both host and viral proteins $^{41}$. In addition, tRNA degradation may also negatively impact the reverse transcription of HIV which requires host tRNA $^{\text {Lys }}$ as the primer ${ }^{42}$.

We observed the preference of SLFN13 for blocking synthesis of viral proteins as compared to endogenous ones. As the key determinant of tRNA cleavage by SLFN13 is the secondary structure but not the anti-codon sequence, we assume that the preference for HIV protein synthesis of SLFN13 may not stem from the codon-usage-based mechanism reported for SLFN $11^{23}$. On the other hand, HIV genome contains rich stem loop regions that are essential for the replication and packaging of the virus ${ }^{43,44}$. SLFN13 may directly digest some of these stem loops to specifically disrupt the life cycle of HIV. The tRNA-derived fragments (tRFs) have been suggested to constitute a new group of functional nonecoding RNAs ${ }^{13}$. These tRFs, generated by certain tRNases including angiogenin, were found to be involved in cellular stress response and cancer progression by means of regulating transcription and translation in cells ${ }^{45-48}$. This mechanism might also apply to the tRNA fragments generated by SLFN13 which retards protein synthesis. Overall, more targets of SLFN13 need to be identified in order to understand its detailed antiviral mechanism.

We found that hSLFN5-N and mSLFN1, albeit both retaining the key catalytic residues (corresponding to Glu205 and Glu210 of rSLFN13), did not cleave the given tRNAs (Fig. 2d). Compared to rSLFN13, hSLFN5 lacks two positively charged residues that were proven critical for tRNA/rRNA digestion (i.e. Lys 42 and Arg217 of rSLFN13), whereas the equivalents of rSLFN13 Arg39, Lys42 and Lys276 are absent in mSLFN1 (Supplementary Fig. 6). In contrast, hSLFN13, mSLFN8, as well as rabbit SLFN14 which was reported active for tRNA/rRNA cleavage ${ }^{24}$, showed higher sequence consistency in the positively charged patches (Fig. 3b; Supplementary Fig. 6). We conclude that the distribution of positively charged residues inside the valley of the $\mathrm{N}^{\prime}$-domain may define the capability and selectivity in tRNA/rRNA cleavage, as well as the antiviral spectrum, for different SLFNs. Although more enzymatic studies and characterisation of the SWADL domain and helicase domain are needed for different SLFNs, it is conceivable that tRNA/rRNA manipulation may be a hallmark of the Schlafen family, and the difference in their $\mathrm{N}^{\prime}$-domains may be one of the factors that define the functional diversity of these proteins.

Regarding the functional diversity of the Schlafen family, other than the restriction of viruses, the inhibition of cell proliferation has been suggested as a prominent character for many members. For example, hSLFN5 was found to negatively regulate the anchorage-independent growth and invasion of malignant melanoma cells ${ }^{19}$. Mouse SLFN1, SLFN2, SLFN3 and SLFN5 were all shown as proliferation suppressors when individually expressed in NIH3T3 murine fibroblasts, T cells or various human tumour cells $^{18,20,25,49-51}$. In addition, mouse SLFN2 was also reported to play an important role in maintaining the quiescence of $\mathrm{T}$ cells and monocytes during immune response ${ }^{52}$. Given the prevalence of the $\mathrm{N}^{\prime}$-domain in SLFNs and the apparent relationship between the translational level and cell proliferation/growth, it is tempting to speculate that SLFNs exert the aforementioned functions through translational regulation. Thus, our study provides valuable clues for investigating the functional mechanisms of the versatile Schlafen family in the future.

\section{Methods}

Molecular cloning. cDNAs of SLFN-N constructs, including those for crystallisation (rSLFN13 ${ }_{14-353}$ ) and cleavage assays (rSLFN13 ${ }_{1-353}$, mSLFN8 $_{1-359}$, hSLFN $13_{1-355}$ and hSLFN5 $5_{12-334}$ ), were individually cloned into a modified pET28 vector. Details of major constructs are illustrated in Supplementary Fig. 1b. For eukaryotic expression, full-length hSLFN13 (hSLFN13 ${ }_{\mathrm{FL}}$ ) and hSLFN13 ${ }_{1-355}$ were individually cloned into the pCAGGS vector with an N-terminal Flag-tag. Indicated mutants were prepared based on corresponding recombinant plasmids by site-directed mutagenesis. In the fluorescence assay, pEGFP-C1 vector was used to generate recombinant plasmid expressing GFP fused hSLFN13 $3_{\mathrm{FL}}$.

Protein expression and purification. Recombinant proteins containing an Nterminal $\mathrm{His}_{6}$-tag followed by a cleavage site for PreScission protease (PSP) were expressed in Escherichia coli (E. coli) Rosetta (DE3). Transformed bacteria were cultured at $37^{\circ} \mathrm{C}$ in Terrific Broth (TB) medium before induced with $0.1 \mathrm{mM}$ isopropyl-1-thio- $\beta$-d-galactopyranoside at an $\mathrm{OD}_{600} \mathrm{~nm}$ of 0.6 , and grown overnight at $16^{\circ} \mathrm{C}$. Cells were lysed in $50 \mathrm{mM}$ Tris- $\mathrm{HCl}, \mathrm{pH} 8.2,300 \mathrm{mM} \mathrm{NaCl}, 30 \mathrm{mM}$ imidazole, $1 \mu \mathrm{M}$ DNase I, $1 \mathrm{mM}$ phenylmethanesulfonylfluoride (PMSF) and $2 \mathrm{mM}$ $\beta$-mercaptoethanol ( $\beta$-ME) using a cell disruptor (JNBIO) and subjected to centrifugation at $40,000 \times g$ for $50 \mathrm{~min}$. The supernatant was filtered and applied to the first Ni-NTA column (GE Healthcare) equilibrated with binding buffer 1 containing $20 \mathrm{mM}$ Tris- $\mathrm{HCl}, \mathrm{pH} 8.2,300 \mathrm{mM} \mathrm{NaCl}, 30 \mathrm{mM}$ imidazole and $2 \mathrm{mM} \beta$ ME. After washed with wash buffer containing $20 \mathrm{mM}$ Tris- $\mathrm{HCl}, \mathrm{pH} 8.2,300 \mathrm{mM}$ $\mathrm{NaCl}, 70 \mathrm{mM}$ imidazole and $2 \mathrm{mM} \beta-\mathrm{ME}$, proteins were eluted with elution buffer containing $20 \mathrm{mM}$ Tris- $\mathrm{HCl}, \mathrm{pH} 8.2,300 \mathrm{mM} \mathrm{NaCl}, 300 \mathrm{mM}$ imidazole and $2 \mathrm{mM}$ $\beta$-ME. Except for rSLFN13 $14-353$, the eluted protein was incubated with glutathione S-transferase (GST)-fused PSP to remove the $\mathrm{His}_{6}$-tag and dialysed overnight against binding buffer 2 containing $20 \mathrm{mM}$ Tris- $\mathrm{HCl}, \mathrm{pH} 8.2,300 \mathrm{mM} \mathrm{NaCl}$ and 2 $\mathrm{mM} \beta$-ME. After dialysis, PSP was removed using a GST column. The protein was re-applied to the second Ni-NTA column equilibrated with binding buffer 2 . Binding buffer 1 was used to elute the proteins which were subsequently loaded onto a Superdex200 16/60 column (GE Healthcare) equilibrated with gel filtration buffer containing $20 \mathrm{mM}$ Tris- $\mathrm{HCl}, \mathrm{pH} 8.2,150 \mathrm{mM} \mathrm{NaCl}$ and $1 \mathrm{mM}$ dithiothreitol (DTT). The proteins were eluted in a discrete peak corresponding to a molecular mass of approximately $40 \mathrm{kDa}$. For rSLFN13 ${ }_{14-353}$, the protein was directly applied to gel filtration using a Superdex200 16/60 column (GE Healthcare) equilibrated with buffer containing $20 \mathrm{mM}$ HEPES, pH 7.0, $150 \mathrm{mM} \mathrm{NaCl}$ and $1 \mathrm{mM}$ DTT after elution from the first Ni-NTA. The proteins were eluted in a discrete peak corresponding to a molecular mass of approximately $80 \mathrm{kDa}$. Cell lysis and protein purification were both performed at $4{ }^{\circ} \mathrm{C}$. The yields of SLFN13-N mutants used for endoribonuclease study were comparable with that of wide-type protein. The selenomethionine (SeMet) derivative of rSLFN13 ${ }_{14-353}$ was insoluble. We therefore made an alanine screening mutagenesis for each methionine of rSLFN13 $3_{14-353}$ to look for soluble constructs, which resulted in $\mathrm{rSLFN}_{13}$ 14-353 $(\mathrm{M} 179 \mathrm{~A})$ as an optimal candidate. SeMet-substituted rSLFN13 ${ }_{14-353}(\mathrm{M} 179 \mathrm{~A})$ was expressed as previously described $^{53}$ and purified as native rSLFN13 $3_{14-353}$, except that $5 \mathrm{mM}$ instead of 2 $\mathrm{mM} \beta$-ME was used in first Ni-NTA purification, and $2 \mathrm{mM}$ instead of $1 \mathrm{mM}$ DTT was used in gel filtration.

Protein crystallisation. Crystallisation trials by the sitting-drop vapour-diffusion method were performed at $4^{\circ} \mathrm{C}$. rSLFN13 $14-353\left(18 \mathrm{mg} \mathrm{ml}^{-1}\right.$ in buffer containing $20 \mathrm{mM}$ HEPES, pH 7.0, $150 \mathrm{mM} \mathrm{NaCl}$ and $1 \mathrm{mM}$ DTT) was mixed with an equal volume of reservoir solution containing 20\% PEG6000, $100 \mathrm{mM} \mathrm{MES,} \mathrm{pH} 6.5$ and $850-950 \mathrm{mM}$ lithium chloride. Crystals of the native protein appeared after 5 days and grew to a maximum size in 10 days. Crystals of SeMet-substituted rSLFN13 ${ }_{14-353}(\mathrm{M} 179 \mathrm{~A})$ were obtained in the same conditions as native rSLFN13 $14-353$. For data collection, crystals of native and Se-Met protein were flash-frozen in the reservoir solution containing extra 20\% PEG200.

Structure determination. X-ray diffraction data set of native rSLFN13 $14-353$ was collected from a single crystal at beamline BL19U1 of the Shanghai Synchrotron Radiation Facility (SSRF), and one data set of SeMet-substituted

rSLFN13 $_{14-353}(\mathrm{M} 176 \mathrm{~A})$ at the selenium peak wavelength was collected at beamline BL17U1 of SSRF ${ }^{54}$. Data sets were processed with the XDS suite ${ }^{55}$ and HKL-2000 programme suite ${ }^{56}$. Initial phases were obtained by the single-anomalous dispersion method and refined using Phaser ${ }^{57}$. In the calculated electron density, the main chains and many side chains were clearly traceable. Owing to the high quality of the density map, an initial model was manually built with COOT $^{58}$, where the positions of 31 selenium atoms in the asymmetric unit were used to assign the sequences of four peptide chains. The model was further refined with $\operatorname{Refmac}^{59}$ and Phenix ${ }^{60}$. Structural validation was carried out using MolProbity ${ }^{61}$. Structural illustrations were prepared using the PyMOL Molecular Graphic Systems (version 0.99, Schrödinger LLC; http://www.pymol.org/). X-ray data collection and refinement statistics can be found in Extended Data Table 1. The Ramachandran statistics determined by PROCHECK ${ }^{62}$ are as follows: $94.48 \%$ in favoured region, $4.97 \%$ allowed, $0.55 \%$ outlier. To confirm the incorporation of zinc, X-ray fluorescence scan over the rSLFN13 $14-353$ crystal was carried out at SSRF, and the excitation energy was $9679 \mathrm{eV}$. 
tRNA substrate preparation. Preparation of tDNA transcription template and in vitro transcription of tRNAs was performed as previously described ${ }^{63}$. To prepare $\left[\alpha^{-32} \mathrm{P}\right]$-labelled tRNA, $250 \mathrm{ng} \mathrm{l}^{-1}$ tDNA transcription template was mixed with $2 \mathrm{mM}$ of each NTP, $3.4 \mu \mathrm{M}\left[\alpha^{-32} \mathrm{P}\right]$ ATP $(6000 \mathrm{Ci} / \mathrm{mmol}$, PerkinElmer), 20 $\mathrm{mM}$ Tris- $\mathrm{HCl}, \mathrm{pH} 8.0,150 \mathrm{mM} \mathrm{NaCl}, 20 \mathrm{mM} \mathrm{MgCl}_{2}, 5 \mathrm{mM}$ DTT, $1 \mathrm{mM}$ spermidine and $0.3 \mu \mathrm{M}$ T7 RNA polymerase at $37^{\circ} \mathrm{C}$ for $1 \mathrm{~h}$. The labelled tRNA product was subsequently purified with a Zymo-Spin IC Column (Zymo Research) and eluted with $200 \mu \mathrm{l}$ TE buffer containing $20 \mathrm{mM}$ Tris- $\mathrm{HCl}, \mathrm{pH} 8.0$ and $1 \mathrm{mM}$ EDTA. The eluted tRNA was aliquoted and stored at $-20^{\circ} \mathrm{C}$ if not immediately used. Sequences of the tRNA transcripts used in this study were listed in Supplementary Table 1.

EMSA. rSLFN $13_{1-353}$ or hSLFN13 $3_{1-355}$ of increasing concentrations was individually incubated with $5 \mu \mathrm{M}$ tRNA ${ }^{\text {Gly(CCC) }}$ or tRNA ${ }^{\text {Ser(UAG) }}$ in buffer containing $40 \mathrm{mM}$ Tris- $\mathrm{HCl}, \mathrm{pH} 8.5,20 \mathrm{mM} \mathrm{KCl}, 2 \mathrm{mM} \mathrm{MgCl} 2,2 \mathrm{mM}$ DTT and 5\% glycerol for $30 \mathrm{~min}$ at $25^{\circ} \mathrm{C}$. After the incubation, each sample was mixed with loading buffer containing $3 \%$ glycerol and $0.05 \%$ bromophenol blue before loaded onto a $6 \%$ native polyacrylamide gel containing $40 \mathrm{mM}$ Tris-acetate, $\mathrm{pH}$ 8.5. The gel was pre-run at $4^{\circ} \mathrm{C}$ for $45 \mathrm{~min}$ at $14.0 \mathrm{~V} \mathrm{~cm}^{-1}$ with running buffer containing $40 \mathrm{mM}$ Tris-acetate, $\mathrm{pH}$ 8.5. After electrophoresis the gel was stained with ethidium bromide (EB) and visualised by UV light.

Cell culture. Unless otherwise specified, HeLa, HEK-293, HEK-293T, Vero and A549 cells were individually maintained at $37^{\circ} \mathrm{C}$ in high glucose Dulbecco's modified Eagle medium (DMEM) supplemented with $10 \%$ fatal calf serum, $100 \mathrm{U}$ $\mathrm{ml}^{-1}$ penicillin and $100 \mu \mathrm{g} \mathrm{ml}^{-1}$ streptomycin. Albopictus C6/36 cells, used for propagation of the Zika virus, were grown in DMEM supplemented with $10 \%$ fetal calf serum at $28^{\circ} \mathrm{C}$. All cell lines are free of mycoplasma. HeLa, 293, 293T and A549 cell lines have been authenticated by genotyping.

In vitro cleavage assay. For substrate screening assay, $2 \mu \mathrm{M}$ rSLFN13 $1-353$ was individually incubated with $1 \mu \mathrm{M}$ each ssDNA, ssRNA, dsDNA, dsRNA, DNA/ RNA duplex, stem-loop RNA or tRNA ${ }^{\text {Ser }}$ in the cleavage buffer containing $40 \mathrm{mM}$ Tris- $\mathrm{HCl}, \mathrm{pH} 8.0,20 \mathrm{mM} \mathrm{KCl}, 4 \mathrm{mM} \mathrm{MgCl}_{2}$ and $2 \mathrm{mM} \mathrm{DTT}$ for $30 \mathrm{~min}$ at $37^{\circ} \mathrm{C}$. The sequences of the nucleic acids used in this experiment are summarised in Supplementary Table 2 .

For cleavage assays on in vitro transcribed tRNAs, either $\left[a-{ }^{32} \mathrm{P}\right]$-labelled or non-labelled tRNA transcripts were used as substrates. For radiolabelled tRNAs to be detected by autoradiography, protein of increasing concentration was mixed with labelled tRNA $\left(>1 \times 10^{4} \mathrm{cpm}\right)$ in the cleavage buffer at $37^{\circ} \mathrm{C}$. Aliquots were taken at different time points as indicated, and the reaction was terminated by adding $2 \times$ formamide gel-loading buffer $(95 \% \mathrm{w} / \mathrm{v}$ formamide, $50 \mathrm{mM}$ EDTA). The cleavage products were analysed by electrophoresis at $250 \mathrm{~V}$ by a $12 \%$ denaturing urea-polyacrylamide gel (Urea-PAGE) containing $7.5 \mathrm{M}$ urea with $1 \times \mathrm{TBE}(89 \mathrm{mM}$ Tris, $89 \mathrm{mM}$ borate and $2 \mathrm{mM}$ EDTA) as the running buffer. All the gels were exposed to a phosphorimager plate overnight before scanned with a Typhoon scanner (GE Healthcare). For non-labelled tRNA substrates, $1 \mu \mathrm{M}$ protein was mixed with $2 \mu \mathrm{M}$ tRNA ${ }^{\text {Ser }}$ in the cleavage buffer at $37^{\circ} \mathrm{C}$. Subsequent experimental procedures were the same as those for labelled tRNA substrates. After electrophoresis, the gel was stained with EB and visualised by UV light.

For cleavage assays on native RNAs, total small RNA was extracted from cultured 293T or HeLa cells using mirVana miRNA Isolation kit (Ambion) following the small RNA isolation protocol. An aliquot of $1 \mu \mathrm{M}$ or indicated

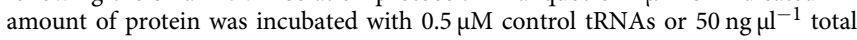
small RNA in the cleavage buffer for $30 \mathrm{~min}$ or indicated time at $37^{\circ} \mathrm{C}$. The cleavage products were analysed by electrophoresis. Total RNA was extracted from cultured 293T or HeLa cells using TRI Reagent (Sigma-Aldrich). Protein of indicated concentrations was incubated with $20 \mathrm{ng} \mathrm{\mu l}^{-1}$ tRNA ${ }^{\mathrm{Ser}}$ control, or $0.4 \mu \mathrm{g}$ $\mu^{-1}$ (for EB staining) or $1 \mu \mathrm{g} \mu \mathrm{l}^{-1}$ (for Northern blot) total RNA in the cleavage buffer for $30 \mathrm{~min}$ at $37^{\circ} \mathrm{C}$. Cleavage products were analysed by electrophoresis, and then either stained with EB or subjected to Northern blot.

Northern blot analysis. The cleavage reaction to be analysed by Northern blot was denatured by heating at $80^{\circ} \mathrm{C}$ for $10 \mathrm{~min}$ in a $2 \times$ formamide gel-loading buffer, followed by quick chill on ice. These samples were separated by Urea-PAGE and subsequently electroblotted onto the Hybond $\mathrm{N}+$ membranes (GE Healthcare) at $250 \mathrm{~mA}$ for $3 \mathrm{~h}$ in $1 \times$ TBE buffer. After air-dried for $10 \mathrm{~min}$, the RNA samples were fixed to the membrane by UV cross-linking. The membrane was pre-hybridised in the hybridisation buffer containing $0.2 \%$ Ficoll $(\mathrm{Mr}=40,000), 0.2 \%$ polyvinylpyrrolidone $(\mathrm{Mr}=40,000), 0.2 \%$ bovine serum albumin (BSA), $50 \%$ formamide, $50 \mathrm{mM}$ Tris- $\mathrm{HCl}, \mathrm{pH} 7.5,1 \mathrm{M} \mathrm{NaCl}, 0.1 \%$ sodium pyrophosphate and $0.1 \mathrm{mg} \mathrm{ml}^{-1}$ denatured salmon sperm DNA at $42^{\circ} \mathrm{C}$ for $3 \mathrm{~h}$. The hybridisation was carried out at $42{ }^{\circ} \mathrm{C}$ overnight in the presence of $\left[\gamma^{32} \mathrm{P}\right]$-ATP $(6000 \mathrm{Ci} / \mathrm{mmol}$, PerkinElmer $)$ DNA oligonucleotide probes which were previously end-labelled using T4 polynucleotide kinase. The probe sequences were complementary to the $5^{\prime}$ end of specified tRNAs and 5S rRNA (see Supplementary Table 3 for details). The hybridised membrane was washed three times with $1 \times$ SSC buffer $(150 \mathrm{mM} \mathrm{NaCl}$, $15 \mathrm{mM}$ sodium citrate, $\mathrm{pH} 7.0$ and $0.1 \% \mathrm{SDS}$ ) at $42^{\circ} \mathrm{C}$ for $30 \mathrm{~min}$ each. The blots were exposed to a phosphorimager plate overnight before scanned with a Typhoon scanner (GE Healthcare).

Western blot analysis. Following antibodies were used in for Western blot assays in this paper: anti-Flag antibody (Sigma-Aldrich, F1804, 1:1000 used), anti- $\beta$ tubulin antibody (ProteinTech, 66240-1-Ig, 1:2000 used), anti-Lamin B1 (ProteinTech, 66095-1-Ig, 1:1000 used), anti-GAPDH (60004-1-Ig, 1:5000 used), HRPconjugated anti-mouse secondary antibody (Cell Signaling Technology, 7076, 1:3000 used) and HRP-conjugated anti-rabbit secondary antibody (ProteinTech, SA00001-2, 1:3000 used). The cell pellets after transfection were lysed in ice-cold lysis buffer (20 mM HEPES, pH 7.9, 1 mM EDTA, $400 \mathrm{mM} \mathrm{NaCl}, 10 \mathrm{mM} \mathrm{KCl}, 1 \%$ Nonidet P-40 and 20\% Glycerol) containing protease inhibitor cocktail and PMSF After centrifugation at $12,000 \times g$ for $15 \mathrm{~min}$, the supernatant separated by $10 \%$ SDS-PAGE, transferred to PVDF membranes (Thermo Scientific). After an $1 \mathrm{~h}$ incubation with blocking buffer ( $5 \%$ BSA), the membrane was incubated with corresponding antibodies overnight at $4{ }^{\circ} \mathrm{C}$. The membrane was washed and incubated with HRP-conjugated secondary antibodies. The membranes were washed again, developed with ECL Western Blotting Detection Reagents (Thermo Scientific) and visualised with ChemiDoc ${ }^{\mathrm{TM}}$ Touch Imaging System (Bio-Rad).

Subcellular localisation analysis. For fluorescence assay, 293T cells and HeLa cells grown on round glass slides in 24-well plates were transfected with pEGFPC1-hSLFN13 ${ }_{\mathrm{FL}}$ using Lipofectamine 2000 (Thermo Scientific). After $24 \mathrm{~h}$, the cells were fixed with $4 \%$ paraformaldehyde (PFA) in phosphate buffered saline (PBS, pH 7.4) at room temperature for $10 \mathrm{~min}$ and washed with PBS for three times. Fixed cells were stained with 4',6-diamidino-2-phenylindole (DAPI, Sigma-Aldrich) for nuclear staining and then mounted in antifade mounting medium (Thermo Scientific). Slides were viewed with an FV1000 fluorescent microscope (Olympus).

For analysis by Western blot, 293T cells were transfected with pCAGGShSLFN13 ${ }_{\mathrm{FL}}$. After $24 \mathrm{~h}$, cells were collected, and NE-PER Nuclear and Cytoplasmic Extraction Reagents (Thermo Scientific) was used to separate nuclear and cytosolic fractions after cell lysis according to manufacturer's instructions. The two fractions were then individually subjected to Western blot analysis.

Quantification of cellular RNA. A total of $1 \times 10^{5} 293 \mathrm{~T}$ cells transfected with empty pCAGGS vector, pCAGGS-hSLFN13 ${ }_{\mathrm{FL}}$ or pCAGGS-hSLFN13 ${ }_{\mathrm{FL}}(\mathrm{E} 213 \mathrm{~A})$ were individually sorted by flow cytometry. Artificial spike-in RNA (1000 nt) was added to the sorted cells as control before total RNA was extracted using TRI Reagent (Sigma-Aldrich). Total RNA was pooled by size and quantified using Bioanalyzer and RNA 6000 Pico Kit (Agilent) following the manufacturer's protocol.

FUNCAT assay and click chemistry. 293T cells transfected with empty pCAGGS vector, pCAGGS-hSLFN13 ${ }_{\mathrm{FL}}$ or pCAGGS-hSLFN13 $\mathrm{FL}(\mathrm{E} 213 \mathrm{~A})$ were re-transfected with corresponding plasmids $12 \mathrm{~h}$ after initial transfection. $36 \mathrm{~h}$ after initial transfection, cells of $80 \%$ confluence were washed twice in pre-warmed PBS and then starved in serum-free DMEM lacking methionine (SFM) for $1 \mathrm{~h}$ (Thermo Scientific). Following the starvation, $4 \mathrm{mM}$ L-HPG (Click Chemistry Tools) or 4 $\mathrm{mM}$ methionine was appended into SFM for a 4 -h pulse as described earlier ${ }^{64}$.

For flow cytometry analysis, the collected cells were fixed in $0.5 \mathrm{ml}$ of $1 \%$ PFA in PBS for $15 \mathrm{~min}$ on ice and permeabilised in PBS containing $0.1 \%$ saponin (SigmaAldrich) for $5 \mathrm{~min}$ at room temperature as described ${ }^{65}$. After labelled by coppercatalysed azide-alkyne cycloaddition (CuAAC), cells were washed and resuspended in PBS, and immediately analysed by flow cytometry. As an additional negative control, $100 \mu \mathrm{g} \mathrm{ml}^{-1}$ translational elongation inhibitor cycloheximide (CHX, Sigma-Aldrich) was added $30 \mathrm{~min}$ before HPG incorporation. Cy5 fluorescence signal was detected using a CytoFLEX flow cytometer (Beckman Coulter).

For SDS-PAGE analysis, cells were collected and lysed in ice-cold lysis buffer (20 mM HEPES, pH 7.9, 1 mM EDTA, $400 \mathrm{mM} \mathrm{NaCl}, 10 \mathrm{mM} \mathrm{KCl}, 1 \%$ Nonidet P 40 and $20 \%$ Glycerol) containing protease inhibitor cocktail and PMSF. Protein concentration was determined using Pierce BCA Protein Assay Kit (Thermo Scientific). HPG incorporated protein lysates were labelled selectively with Cy5conjugated azide by CuAAC ${ }^{66,67}$. The click reaction was performed following the protocol from Click Chemistry Protein Reaction Buffer Kit (Click Chemistry Tools): $70 \mu \mathrm{l}$ soluble protein lysates $\left(1.5-4 \mu \mathrm{g} \mu \mathrm{l}^{-1}\right)$ incorporated with HPG or methionine were sequentially mixed with $90 \mu \mathrm{l}$ Reaction Buffer, $1 \mu \mathrm{l}$ Cy5-azide (8 $\mathrm{mM}), 10 \mu \mathrm{l}$ Tris(3-hydroxypropyltriazolylmethyl)amine (THPTA, $100 \mathrm{mM}$ ), $10 \mu \mathrm{l}$ $\mathrm{CuSO}_{4}(20 \mathrm{mM})$ and $10 \mu \mathrm{l}$ sodium ascorbate $(300 \mathrm{mM})$. The mixture was rotated end-over-end in dark for $30 \mathrm{~min}$ at room temperature to generate a stable triazole adduct, and then subjected to $1 \times$ Laemmli buffer. After heated at $70{ }^{\circ} \mathrm{C}$ for $10 \mathrm{~min}$ the samples were resolved by SDS-PAGE and imaged using a pharos $\mathrm{FX}^{\mathrm{TM}}$ Plus Molecular Imager (Bio-Rad). Loading consistency was confirmed by staining the gels with Coomassie Blue after fluorescent imaging.

Assay for HIV, HSV and Zika virus production. All HIV-related assays in this study were based on HIV-1. To analyse HIV retrovirus production, pNL4-3- $\Delta$ EnvEGFP vector and pCMV-VSV-G packaging vector were transfected into the 293T cells which were pre-transfected with empty pCAGGS vector, pCAGGShSLFN13 ${ }_{\mathrm{FL}}$, pCAGGS-hSLFN13 ${ }_{1-355}$ or corresponding mutants using 
Lipofectamine 2000 (Thermo Scientific) according to the manufacturer's instructions. The old culture medium was replaced with fresh culture medium at $6 \mathrm{~h}$ posttransfection. Subsequently, the supernatants were collected at $48 \mathrm{~h}$ posttransfection and cleared by centrifugation. The amount of (infectious) virus particles in the supernatants was determined via infection assay, quantitative (q)PCR of viral RNA (total RNA and unspliced RNA) and HIV p24 ELISA (Supplementary Table 4). For infection assays, pre-plated $293 \mathrm{~T}$ cells were infected with serially diluted supernatants for $6 \mathrm{~h}$. At $24 \mathrm{~h}$ post-infection, the 293T cells were examined for GFP expression by flow cytometry. HIV viral RNA was extracted from the virus supernatants and quantified. The amount of p24 was measured using an HIV-1 p24 ELISA Kit (Clonetech) according to the manufacturer's instructions. The expression of intracellular GFP was measured by flow cytometry.

For HIV package assays in 293 cells, pNL4-3- $\Delta$ Env-EGFP vector and pCMVVSV-G packaging vector were transfected into the 293 cells which were pretransfected with scramble-siRNA, SLFN13-siRNA\#1 or SLFN13-siRNA\#2. The supernatants were collected at $48 \mathrm{~h}$ post-transfection and measured by infecting pre-plated $293 \mathrm{~T}$ cells. The mRNA level of hSLFN13 was measured by qPCR (primers are listed in Supplementary Table 4).

The GFP fused HSV is a gift from Prof. G. F. Gao. For virus preparation, preplated Vero cells were infected with HSV $(\mathrm{MOI}=0.03)$ in serum-free DMEM for 2 h. $48-72 \mathrm{~h}$ after the infection, the Vero cells became suspension and were collected. The virus-containing lysates were generated by freeze/thaw cycles followed by centrifugation at $4000 \mathrm{rpm}$ for $15 \mathrm{~min}$. For HSV infection and production assay, pre-plated 293T cells were infected with HSV $(\mathrm{MOI}=0.02)$ after transfected with pCAGGS-hSLFN13 and related mutants for $2 \mathrm{~h}$. The supernatants were collected $18 \mathrm{~h}$ after infection and diluted to infect pre-plated Vero cells for plaque formation and HSV titre determination.

For Zika virus production, albopictus C6/36 cells were infected with wide-type strain MR766 at $28^{\circ} \mathrm{C}$ and virus supernatants were collected 5 days after infection. For Zika infection and production assay, A549 cells overexpressing pCAGGShSLFN13 and corresponding mutants were rinsed once with PBS, and infected with virus for $2 \mathrm{~h}$ at $37^{\circ} \mathrm{C} .48 \mathrm{~h}$ after infection, the supernatants were collected and diluted to infect pre-plated Vero cells for plaque assay.

Assay for HIV infection. HIV VSV-G was prepared by transfecting 293T cells with pNL4-3- $\triangle$ Env-EGFP and pCMV-VSV-G using Lipofectamine 2000 (Thermo Scientific). The cell culture medium was collected and cleared as described above. Cells to be tested were infected for $6 \mathrm{~h}$. At $24 \mathrm{~h}$ post-infection, GFP expression was measured to determine the relative infection efficiencies.

Determination of viral RNA level. Virion supernatants were lysed and homogenised with TRIzol Reagent (Thermo Scientific) following the manufacturer's protocol. Viral protein and DNA were removed by centrifugation at $4{ }^{\circ} \mathrm{C}$ for 15 min at $12,000 \times g$, and vRNA was extracted from the upper aqueous phase and subsequently reverse transcribed using PrimeScript RT reagent Kit (TaKaRa). The concentrations of total vRNA and unspliced vRNA were individually determined by qPCR with CFX96 Real-Time System (Bio-Rad) using SYBR Premix ExTaq Kit (TaKaRa). Primers used in qPCR are listed in Supplementary Table 4.

Data availability. The X-ray crystallographic coordinates and structure factor files for rSLFN13-N has been deposited in the Protein Data Bank (PDB) under accession number 5YD0. All other data generated or analysed during this study are included in this published article, and are available from the corresponding author upon reasonable request.

Received: 14 December 2017 Accepted: 20 February 2018

Published online: 21 March 2018

\section{References}

1. Holcik, M. \& Sonenberg, N. Translational control in stress and apoptosis. Nat. Rev. Mol. Cell. Biol. 6, 318-327 (2005).

2. McCormick, C. \& Khaperskyy, D.A. Translation inhibition and stress granules in the antiviral immune response. Nat. Rev. Immunol. 17, 647-660 (2017).

3. Robichaud, N. \& Sonenberg, N. Translational control and the cancer cell response to stress. Curr. Opin. Cell. Biol. 45, 102-109 (2017).

4. Jackson, R. J., Hellen, C. U. \& Pestova, T. V. The mechanism of eukaryotic translation initiation and principles of its regulation. Nat. Rev. Mol. Cell. Biol. 11, 113-127 (2010)

5. Anderson, P. \& Kedersha, N. RNA granules: post-transcriptional and epigenetic modulators of gene expression. Nat. Rev. Mol. Cell. Biol. 10, 430-436 (2009).

6. Sofos, N., Xu, K., Dedic, E. \& Brodersen, D. E. Cut to the chase-Regulating translation through RNA cleavage. Biochimie 114, 10-17 (2015).
7. Thompson, D. M., Lu, C., Green, P. J. \& Parker, R. tRNA cleavage is a conserved response to oxidative stress in eukaryotes. RNA 14, 2095-2103 (2008).

8. Thompson, D. M. \& Parker, R. Stressing out over tRNA cleavage. Cell 138, 215-219 (2009).

9. Masaki, H. \& Ogawa, T. The modes of action of colicins E5 and D, and related cytotoxic tRNases. Biochimie 84, 433-438 (2002).

10. Cruz, J.W. \& Woychik, N.A. tRNAs taking charge. Pathog. Dis. 74, ftv117 (2016).

11. Haussecker, D. et al. Human tRNA-derived small RNAs in the global regulation of RNA silencing. RNA 16, 673-695 (2010).

12. Gebetsberger, J. \& Polacek, N. Slicing tRNAs to boost functional ncRNA diversity. RNA Biol. 10, 1798-1806 (2013).

13. Lee, Y. S., Shibata, Y., Malhotra, A. \& Dutta, A. A novel class of small RNAs: tRNA-derived RNA fragments (tRFs). Genes Dev. 23, 2639-2649 (2009).

14. Thompson, D. M. \& Parker, R. The RNase Rnylp cleaves tRNAs and promotes cell death during oxidative stress in Saccharomyces cerevisiae. J. Cell. Biol. 185, 43-50 (2009).

15. Fu, H. et al. Stress induces tRNA cleavage by angiogenin in mammalian cells FEBS Lett. 583, 437-442 (2009).

16. Schwarz, D. A., Katayama, C. D. \& Hedrick, S. M. Schlafen, a new family of growth regulatory genes that affect thymocyte development. Immunity $\mathbf{9}$, 657-668 (1998)

17. Bustos, O. et al. Evolution of the Schlafen genes, a gene family associated with embryonic lethality, meiotic drive, immune processes and orthopoxvirus virulence. Gene 447, 1-11 (2009).

18. Katsoulidis, E. et al. Role of Schlafen 2 (SLFN2) in the generation of interferon alpha-induced growth inhibitory responses. J. Biol. Chem. 284, 25051-25064 (2009).

19. Katsoulidis, E. et al. Role of interferon \{alpha\} (IFN $\{a l p h a\}$ )-inducible Schlafen-5 in regulation of anchorage-independent growth and invasion of malignant melanoma cells. J. Biol. Chem. 285, 40333-40341 (2010).

20. Mavrommatis, E. et al. Expression and regulatory effects of murine Schlafen (Slfn) genes in malignant melanoma and renal cell carcinoma. J. Biol. Chem. 288, 33006-33015 (2013).

21. Seong. R.K. et al. Schlafen 14 (SLFN14) is a novel antiviral factor involved in the control of viral replication. Immunobiology 222, 979-988 (2017).

22. Mavrommatis, E., Fish, E. N. \& Platanias, L. C. The schlafen family of proteins and their regulation by interferons. J. Interferon Cytokine Res. 33, 206-210 (2013).

23. Li, M. et al. Codon-usage-based inhibition of HIV protein synthesis by human schlafen 11. Nature 491, 125-128 (2012).

24. Pisareva, V. P., Muslimov, I. A., Tcherepanov, A. \& Pisarev, A. V. Characterization of novel ribosome-associated endoribonuclease SLFN14 from rabbit reticulocytes. Biochemistry 54, 3286-3301 (2015).

25. Geserick, P., Kaiser, F., Klemm, U., Kaufmann, S. H. \& Zerrahn, J. Modulation of $\mathrm{T}$ cell development and activation by novel members of the Schlafen (slfn) gene family harbouring an RNA helicase-like motif. Int. Immunol. 16, 1535-1548 (2004)

26. Li, H., Trotta, C. R. \& Abelson, J. Crystal structure and evolution of a transfer RNA splicing enzyme. Science 280, 279-284 (1998).

27. Lin, Y. L., Elias, Y. \& Huang, R. H. Structural and mutational studies of the catalytic domain of colicin E5: a tRNA-specific ribonuclease. Biochemistry $\mathbf{4 4}$ 10494-10500 (2005).

28. Acharya, K. R., Shapiro, R., Riordan, J. F. \& Vallee, B. L. Crystal structure of bovine angiogenin at 1.5-A resolution. Proc. Natl Acad. Sci. USA 92, 2949-2953 (1995).

29. Chakravarty, A. K., Smith, P., Jalan, R. \& Shuman, S. Structure, mechanism, and specificity of a eukaryal tRNA restriction enzyme involved in self-nonself discrimination. Cell Rep. 7, 339-347 (2014).

30. Kwon, S. C. et al. Structure of human DROSHA. Cell 164, 81-90 (2016).

31. Macrae, I. J. et al. Structural basis for double-stranded RNA processing by Dicer. Science 311, 195-198 (2006).

32. Holm, L. \& Rosenstrom, P. Dali server: conservation mapping in 3D. Nucleic Acids Res. 38, W545-W549 (2010).

33. Callaghan, A. J. et al. Structure of Escherichia coli RNase E catalytic domain and implications for RNA turnover. Nature 437, 1187-1191 (2005).

34. Gan, J. et al. Structural insight into the mechanism of double-stranded RNA processing by ribonuclease III. Cell 124, 355-366 (2006).

35. Yamasaki, S., Ivanov, P., Hu, G. F. \& Anderson, P. Angiogenin cleaves tRNA and promotes stress-induced translational repression. J. Cell Biol. 185, 35-42 (2009).

36. Levitz, R. et al. The optional E. coli prr locus encodes a latent form of phage T4-induced anticodon nuclease. EMBO J. 9, 1383-1389 (1990).

37. Ogawa, T. et al. A cytotoxic ribonuclease targeting specific transfer RNA anticodons. Science 283, 2097-2100 (1999). 
38. Tomita, K., Ogawa, T., Uozumi, T., Watanabe, K. \& Masaki, H. A cytotoxic ribonuclease which specifically cleaves four isoaccepting arginine tRNAs at their anticodon loops. Proc. Natl Acad. Sci. USA 97, 8278-8283 (2000).

39. Morse, R. P. et al. Structural basis of toxicity and immunity in contactdependent growth inhibition (CDI) systems. Proc. Natl Acad. Sci. USA 109, 21480-21485 (2012).

40. Jones, A. M., Garza-Sanchez, F., So, J., Hayes, C. S. \& Low, D. A. Activation of contact-dependent antibacterial tRNase toxins by translation elongation factors. Proc. Natl Acad. Sci. USA 114, E1951-E1957 (2017).

41. Stabell, A. C. et al. Non-human primate schlafen11 inhibits production of both host and viral proteins. PLoS Pathog. 12, e1006066 (2016).

42. Ratner, L. et al. Complete nucleotide sequences of the AIDS virus, HTLV-III. Nature 313, 277-284 (1985)

43. Malim, M. H. et al. HIV-1 structural gene expression requires binding of the Rev trans-activator to its RNA target sequence. Cell 60, 675-683 (1990).

44. Clever, J. L., Eckstein, D. A. \& Parslow, T. G. Genetic dissociation of the encapsidation and reverse transcription functions in the $5^{\prime} \mathrm{R}$ region of human immunodeficiency virus type 1. J. Virol. 73, 101-109 (1999).

45. Ivanov, P., Emara, M. M., Villen, J., Gygi, S. P. \& Anderson, P. Angiogenininduced tRNA fragments inhibit translation initiation. Mol. Cell. 43, 613-623 (2011).

46. Goodarzi, H. et al. Endogenous tRNA-derived fragments suppress breast cancer progression via YBX1 displacement. Cell 161, 790-802 (2015)

47. Huang, B. et al. tRF/miR-1280 suppresses stem cell-like cells and metastasis in colorectal cancer. Cancer Res. 77, 3194-3206 (2017).

48. Anderson, P. \& Ivanov, P. tRNA fragments in human health and disease. FEBS Lett. 588, 4297-4304 (2014).

49. Brady, G., Boggan, L., Bowie, A. \& O’Neill, L. A. Schlafen-1 causes a cell cycle arrest by inhibiting induction of cyclin D1. J. Biol. Chem. 280, 30723-30734 (2005).

50. Patel, B. B. et al. Schlafen 3, a novel gene, regulates colonic mucosal growth during aging. Am. J. Physiol. Gastrointest. Liver. Physiol. 296, G955-G962 (2009).

51. Sassano, A. et al. Human schlafen 5 (SLFN5) Is a regulator of motility and invasiveness of renal cell carcinoma cells. Mol. Cell. Biol. 35, 2684-2698 (2015).

52. Berger, M. et al. An Slfn2 mutation causes lymphoid and myeloid immunodeficiency due to loss of immune cell quiescence. Nat. Immunol. 11, 335-343 (2010).

53. Cao, Y. L. et al. MFN1 structures reveal nucleotide-triggered dimerization critical for mitochondrial fusion. Nature 542, 372-376 (2017).

54. Wang, Q. S. et al. The macromolecular crystallography beamline of SSRF. Nucl. Sci. Tech. 26, 12-17 (2015).

55. Kabsch, W. XDS. Acta Crystallogr. D Biol. Crystallogr. 66, 125-132 (2010).

56. Otwinowski, Z. \& Minor, W. Processing of X-ray diffraction data collected in oscillation mode. Methods Enzymol. 276, 307-326 (1997).

57. McCoy, A. J. et al. Phaser crystallographic software. J. Appl. Cryst. 40, 658-674 (2007).

58. Emsley, P. \& Cowtan, K. Coot: model-building tools for molecular graphics. Acta Crystallogr. D Biol. Crystallogr. 60, 2126-2132 (2004). Pt 12 Pt 1.

59. Murshudov, G. N., Vagin, A. A. \& Dodson, E. J. Refinement of macromolecular structures by the maximum-likelihood method. Acta Crystallogr. D Biol. Crystallogr. 53, 240-255 (1997)

60. Adams, P. D. et al. PHENIX: a comprehensive Python-based system for macromolecular structure solution. Acta Crystallogr. D Biol. Crystallogr. 66 213-221 (2010).

61. Chen, V. B. et al. MolProbity: all-atom structure validation for macromolecular crystallography. Acta Crystallogr. D Biol. Crystallogr. 66, 12-21 (2010).

62. Laskowski, R. A., MacArthur, M. W., Moss, D. S. \& Thornton, J. M. PROCHECK: a program to check the stereochemical quality of protein structures. J. Appl. Cryst. 26, 283-291 (1993).

63. Qin, X. et al. Cocrystal structures of glycyl-tRNA synthetase in complex with tRNA suggest multiple conformational states in glycylation. J. Biol. Chem. 289, 20359-20369 (2014)
64. Beatty, K. E. et al. Fluorescence visualization of newly synthesized proteins in mammalian cells. Angew. Chem. 45, 7364-7367 (2006).

65. Sendoel, A. et al. Translation from unconventional 5' start sites drives tumour initiation. Nature 541, 494-499 (2017).

66. McShane, E. et al. Kinetic analysis of protein stability reveals age-dependent degradation. Cell 167, 803-815 (2016). e821.

67. Calve, S., Witten, A. J., Ocken, A. R. \& Kinzer-Ursem, T. L. Incorporation of non-canonical amino acids into the developing murine proteome. Sci. Rep. 6 , 32377 (2016).

\section{Acknowledgements}

We thank the staff at beamline BL17U1 and BL19U1 of SSRF for the help with the collection of diffraction data; G. Kochs and T. Pan for sharing plasmids used for this study; C. Lavender for discussion on the manuscript. This work was supported by grants of National Natural Science Foundation of China (81772977, 31722016 and 31470729), National Basic Research Program of China (2013CB910500), Natural Science Foundation of Guangdong Province (2014TQ01R584 and 2014A030312015), New Century Excellen Talents in University (NCET-12-0567) and the Recruitment Program of Global Youth Experts to S.G., and Fundamental Research Funds for the Central Universities (16lgjc76) to W.X.

\section{Author contributions}

S.G., W.X. and J.-Y.Y. conceived the project. J.-Y.Y. solved the structure together with S. G., and carried out most functional assays. X.-Y.D. performed tRNA cleavage assays. Y.S.L., X.W. and W.C. did in vivo tRNA and rRNA turnover assays. X.-C.M. and H.Z. conducted HIV titre and infection assays. J.-X.F., B.Y. and Y.C. helped with molecular cloning, protein purification and diffraction data collection. L.-Y.L., M.-L.C., Z.-X.F., Q. Z., T.-B.K., L.-B.S. and M.-S.Z. helped with cell culture and fluorescence assays. F.-X.Z. Y.-P.L. and R.-H.X. contributed to the analysis of preliminary data. S.G., W.X., J.-Y.Y. and X.-Y.D. wrote the paper. All authors contributed to the discussion on the manuscript.

\section{Additional information}

Supplementary Information accompanies this paper at https://doi.org/10.1038/s41467018-03544-x.

Competing interests: The authors declare no competing interests.

Reprints and permission information is available online at http://npg.nature.com/ reprintsandpermissions/

Publisher's note: Springer Nature remains neutral with regard to jurisdictional claims in published maps and institutional affiliations.

Open Access This article is licensed under a Creative Commons Attribution 4.0 International License, which permits use, sharing, adaptation, distribution and reproduction in any medium or format, as long as you give appropriate credit to the original author(s) and the source, provide a link to the Creative Commons license, and indicate if changes were made. The images or other third party material in this article are included in the article's Creative Commons license, unless indicated otherwise in a credit line to the material. If material is not included in the article's Creative Commons license and your intended use is not permitted by statutory regulation or exceeds the permitted use, you will need to obtain permission directly from the copyright holder. To view a copy of this license, visit http://creativecommons.org/ licenses/by/4.0/.

(C) The Author(s) 2018 